\title{
Development of New Thermodynamic Cycles
}

D. Yogi Goswami

\author{
Professor and Director
}

Solar Energy and Energy Conversion Laboratory

University of Florida 


\section{CHAPTER 4 \\ OPTIMIZATION OF AMMONIA-BASED COMBINED POWER/COOLING CYCLE}

In general, optimization is a way to find the best solution to a problem of any kind. We do a lot of optimization in our daily life. For example, we always search for the best product, which meets our demands, with the lowest possible price. In engineering, optimization is a very powerful tool. There is usually more than one acceptable solution to an engineering problem. The goal of an engineer is to find the best one. When there are more than a few acceptable solutions, it may be simply impossible to compare them by hand. More sophisticated mathematical methods have been developed to help find the best solution.

\section{Introduction to Optimization}

\section{Mathematical Formulation}

Mathematically speaking, optimization is the minimization or maximization of a function subject to a set of constraints on its variables. For a thermodynamic cycle, the optimization objective usually is to find the maximum efficiency or work output. Constraints would be like approach temperatures in a heat exchanger. Engineering optimization problems, despite their diversity, have amazingly the same mathematical formulation. It can be written as: 


$$
\begin{array}{lc}
\min & f(\boldsymbol{x}) \\
\text { s.t. } & \boldsymbol{h}(\boldsymbol{x})=0 \\
& \boldsymbol{g}(\boldsymbol{x}) \leq 0
\end{array}
$$

Where

$\boldsymbol{x}$ is the vector of a set of continuous real variables;

$f$ is the objective function, a function of $x$ that we want to minimize;

$\boldsymbol{h}(\boldsymbol{x})=0$ and $\boldsymbol{g}(\boldsymbol{x}) \leq 0$ are sets of equality constraints and inequality constraints, respectively.

If an optimization problem is to maximize rather than minimize $f$, we can easily accommodate this change by minimizing $-f$ in the formulation (4.1). A well-defined optimization problem features a number of variables greater than the number of equality constraints, which implies that there exist degrees of freedom upon which we optimize.

Formulation (4.1) contains a number of classes of optimization problems, by appropriate consideration or elimination of its elements. If a problem doesn't have constraints on the variables, then formulation (4.1) becomes an unconstrained optimization problem. Otherwise it's a constrained optimization problem. Among constrained optimization problems, if both the objective function and all the constraints are linear functions of $\boldsymbol{x}$, then formulation (4.1) becomes a linear programming (LP) problem. If at least one of the constraints or the objective function is nonlinear function, formulation (4.1) becomes a nonlinear programming (NLP) problem. A special case of constrained optimization is bound-constrained optimization. In this special case, all constraints are boundary constraints on $\boldsymbol{x}(\boldsymbol{L} \leq \boldsymbol{x} \leq \boldsymbol{U})$. 


\section{Optimality Conditions}

Before we start to search for the optimum, we need to answer a very basic question. How do we tell if a point is an optimum or not? Apparently, a condition or conditions are required to make such judgement. That condition or conditions are termed as optimality conditions. This section presents the necessary and sufficient optimality conditions for unconstrained and constrained optimization.

\section{Unconstrained optimization}

An unconstrained optimization problem deals with the search for a minimum of a function $f(x)$ of $\mathrm{n}$ real variables $\boldsymbol{x}=\left(x_{1}, x_{2}, \ldots, x_{\mathrm{n}}\right)$. For an unconstrained optimization problem, formulation (4.1) is simplified as:

$\min f(x)$

Each of the real variables $x_{i}$ is allowed to take any value from $-\infty$ to $\infty$.

Unconstrained optimization plays an important role in the optimization applications. It is not only because there are some applications which inherently are unconstrained, but also because constrained optimization algorithms are often the extensions of unconstrained optimization algorithms. The necessary and sufficient optimality conditions for an unconstrained optimization problem are:

\section{Theorem 4.1}

Let $f(x)$ be a twice differentiable function in $R^{n}$ at $x^{*}$. Then $x^{*}$ is a local minimum if and only if 
i. $\nabla f\left(x^{*}\right)=0$, and

ii. The Hessian matrix $H\left(x^{*}\right)$, given by

$$
H\left(x^{*}\right)=\frac{\partial^{2} f}{\partial x_{i} \partial x_{j}}\left(x^{*}\right)
$$

is positive semidefinite; that is, $y^{T} H\left(x^{*}\right) y \geq 0$ for all $y \in R^{n}$.

When there's only one variable, the above necessary and sufficient conditions are simplified into a more familiar format:

i. $f^{\prime}(x)=0$;

ii. $f^{\prime \prime}(x) \geq 0$.

\section{Constrained optimization}

A constrained optimization problem deals with the search for a minimum of a function $f(x)$ of $\mathbf{n}$ real variables $\boldsymbol{x}=\left(x_{1}, x_{2}, \cdots, x_{\mathrm{n}}\right)$ subject to a set of equality constraints $\boldsymbol{h}(\boldsymbol{x})=0\left(h_{i}(\boldsymbol{x})=0, i=1,2, \cdots, m\right)$, and a set of inequality constraints $\boldsymbol{g}(\boldsymbol{x}) \leq 0\left(g_{j}(\boldsymbol{x}) \leq 0\right.$ $j=1,2, \cdots, p)$. It has the same form of formulation (4.1), which is rewritten here:

$$
\begin{array}{ll}
\min & f(x) \\
\text { s.t. } & \boldsymbol{h}(\boldsymbol{x})=0 \\
& \boldsymbol{g}(\boldsymbol{x}) \leq 0
\end{array}
$$

If a point $\bar{x}$ satisfies the equality and inequality constraints in formulation (4.1), then it is a feasible point of the problem. Among inequality constraints, an inequality constraint $g_{j}(x)$ is called active at a feasible point $\bar{x}$ if $g_{j}(\bar{x})=0$ and inactive if $g_{j}(\bar{x})<0$. 
Compared with unconstrained optimization, constrained optimization is much more complicated. The key idea in developing necessary and sufficient optimality conditions for constrained optimization problems is to transform them into unconstrained problems and then apply the optimality conditions of unconstrained problems (Nocedal and Wright, 1999). The transformation involves the introduction of an auxiliary function, called the Lagrange function $L(x)$, defined as

$$
L(\boldsymbol{x}, \lambda, \boldsymbol{\mu})=f(\boldsymbol{x})+\lambda^{T} \boldsymbol{h}(\boldsymbol{x})+\boldsymbol{\mu}^{T} \boldsymbol{g}(\boldsymbol{x}), \quad \boldsymbol{\mu} \geq 0,
$$

Where $\lambda^{T}=\left(\lambda_{1}, \lambda_{2}, \cdots, \lambda_{m}\right)$ and $\mu^{T}=\left(\mu_{1}, \mu_{2}, \cdots, \mu_{p}\right)$ are the Lagrange multipliers associated with the equality and inequality constraints, respectively. The multipliers $\lambda$ can be any real numbers, while the multipliers $\mu$ must be nonnegative.

Lagrange multipliers in formulation (4.4) are not just a group of coefficients. They have an important special meaning in constrained optimization. Each Lagrange multiplier for a given constraint indicates how much the Lagrange function $L$ will change for a differential change of that constraint. Considering a simple problem with only one equality constraint:

$$
\begin{array}{ll}
\min & f(x) \\
\text { s.t. } & h(x)=e
\end{array}
$$

Then its Lagrange function $L(\boldsymbol{x}, \lambda)$ is:

$$
L(x, \lambda)=f(x)+\lambda[h(x)-e]
$$

Take a derivative of the Lagrange function $L(x, \lambda)$ with respect to $e$ :

$$
\frac{\partial L}{\partial e}=-\lambda
$$


Therefore, the change of $L$ with the constant e is proportional to the Lagrange multiplier. Since the Lagrange function $L$ is equal to the objective function $f$ at the optimum, we can say that the Lagrange multipliers indicate the sensitivity of the objective function $f(x)$ with respect to perturbations on the constraints.

With the introduction of Lagrange function, a constrained optimization problem is transformed into an unconstrained problem according to duality theory (Floudas, 1995):

$$
\min _{x, \lambda, \mu \geq 0} L(\boldsymbol{x}, \lambda, \mu)=f(\boldsymbol{x})+\lambda^{T} \boldsymbol{h}(\boldsymbol{x})+\boldsymbol{\mu}^{T} \boldsymbol{g}(\boldsymbol{x})
$$

The necessary optimality conditions are given as below:

\section{Theorem 4.2 Karush-Kuhn-Tucker Necessary Conditions}

Let $f(\boldsymbol{x})$ and $\boldsymbol{g}(\boldsymbol{x})$ be differentiable at $\boldsymbol{x}^{*}$ and $\boldsymbol{h}(\boldsymbol{x})$ have continuous first partial derivatives at $x^{*}$. If $x^{*}$ is a local optimum of problem (4.1) and one constraint qualification ${ }^{1}$ is satisfied. Then there exist Lagrange multipliers $\lambda, \mu$ :

$$
\begin{aligned}
& \nabla f\left(\boldsymbol{x}^{*}\right)+\lambda^{T} \nabla \boldsymbol{h}\left(\boldsymbol{x}^{*}\right)+\mu^{T} \nabla \boldsymbol{g}\left(\boldsymbol{x}^{*}\right)=0 \\
& \boldsymbol{h}\left(\boldsymbol{x}^{*}\right)=0 \\
& \boldsymbol{g}\left(\boldsymbol{x}^{*}\right) \leq 0 \\
& \mu_{j} g_{j}\left(\boldsymbol{x}^{*}\right)=0 \quad j=1,2, \cdots, p \\
& \mu_{j} \geq 0 \quad j=1,2, \cdots, p .
\end{aligned}
$$

A point which satisfies Karush-Kuhn-Tucker conditions is called a KKT point.

\footnotetext{
${ }^{1}$ First-order and second-order constraint qualifications are satisfied if the gradients of all equality and active inequality constraints are linearly independent.
} 
However, theorem 4.2 only utilizes the first-order information of the objective function and constraints. The second order information, the curvature of the functions, is not considered. Fiacco and McCormick (1968) demonstrated that first-order information is not complete with their famous example:

$$
\begin{aligned}
& \min \quad\left(x_{1}-1\right)^{2}+x_{2}{ }^{2} \\
& \text { s.t. } \quad x_{1}-\frac{x_{2}^{2}}{k} \leq 0
\end{aligned}
$$

Where the values of the parameter $k>0$, for which $(0,0)$ is a local minimum, are sought.

In this example, we only have one constraint. Its gradient

$$
\left(1,-\frac{2 x_{2}}{k}\right)
$$

is always nonzero. So it's linearly independent by itself. A constrain qualification is satisfied. At $(0,0)$, the gradient KKT conditions are:

$$
\begin{aligned}
& \left(\begin{array}{c}
-2 \\
0
\end{array}\right)+\mu\left(\begin{array}{l}
1 \\
0
\end{array}\right)=\left(\begin{array}{l}
0 \\
0
\end{array}\right) \\
& \therefore \quad \mu=2 \quad \geq 0
\end{aligned}
$$

The constraints KKT conditions are satisfied since:

$$
x_{1}-\frac{x_{2}^{2}}{k}=0
$$

Therefore, KKT necessary conditions are satisfied. However, for $k=1$ the point $(0,0)$ is not a local minimum while for $k=4$ it is.

From the above example, we learn that in order to find a local optimum, second order information has to be taken into account. The second order necessary and sufficient conditions are presented: 


\section{Theorem 4.3 Second-Order Necessary Optimality Conditions}

Let $f(\boldsymbol{x}), \boldsymbol{h}(\boldsymbol{x})$ and $\boldsymbol{g}(\boldsymbol{x})$ be twice continuously differentiable at $\boldsymbol{x}^{*}$. If $\boldsymbol{x}^{*}$ is a local optimum of problem (4.1) and the second order constraint qualification is satisfied ${ }^{1}$, and if there exist Lagrange multipliers $\lambda^{*}, \mu^{*}$ satisfying the KKT first-order necessary conditions:

$$
\begin{aligned}
\nabla f\left(\boldsymbol{x}^{*}\right)+\lambda *^{T} \nabla \boldsymbol{h}\left(\boldsymbol{x}^{*}\right)+\mu^{* T} \nabla \boldsymbol{g}\left(\boldsymbol{x}^{*}\right) & =0 \\
\boldsymbol{h}\left(\boldsymbol{x}^{*}\right) & =0 \\
\boldsymbol{g}\left(\boldsymbol{x}^{*}\right) & \leq 0 \\
\mu_{j}^{*} g_{j}\left(\boldsymbol{x}^{*}\right) & =0 \quad j=1,2, \cdots, p \\
\mu_{j}^{*} & \geq 0 \quad j=1,2, \cdots, p
\end{aligned}
$$

and if for every nonzero vector $z$ :

$$
\begin{array}{cc}
z^{T} \nabla h_{i}\left(x^{*}\right)=0 & i=1,2, \cdots, m, \text { and } \\
z^{T} \nabla g_{i}\left(x^{*}\right)=0 & j \in J \equiv\left\{j: g_{j}\left(x^{*}\right)=0\right\}
\end{array}
$$

then,

$$
z^{T} \nabla^{2} L\left(x^{*}, \lambda^{*}, \mu^{*}\right) z \geq 0
$$

\section{Theorem 4.4 Second-Order Sufficient Optimality Conditions}

Let $f(\boldsymbol{x}), \boldsymbol{h}(\boldsymbol{x})$ and $\boldsymbol{g}(\boldsymbol{x})$ be twice differentiable at $\boldsymbol{x}^{*}$. If there exist Lagrange multipliers $\lambda^{*}, \mu^{*}$ satisfying the KKT first-order necessary conditions:

$$
\begin{aligned}
\nabla f\left(\boldsymbol{x}^{*}\right)+\lambda^{* T} \nabla \boldsymbol{h}\left(\boldsymbol{x}^{*}\right)+\boldsymbol{\mu}^{*^{T}} \nabla \boldsymbol{g}\left(\boldsymbol{x}^{*}\right) & =0 \\
\boldsymbol{h}\left(\boldsymbol{x}^{*}\right) & =0
\end{aligned}
$$




$$
\begin{array}{r}
\boldsymbol{g}\left(\boldsymbol{x}^{*}\right) \leq 0 \\
\mu_{j}^{*} g_{j}\left(x^{*}\right)=0 \quad j=1,2, \cdots, p \\
\mu_{j}^{*} \geq 0 \quad j=1,2, \cdots, p
\end{array}
$$

If for every nonzero vector $z$ :

$$
\begin{array}{ll}
z^{T} \nabla h_{i}\left(x^{*}\right)=0 & i=1,2, \cdots, m, \text { and } \\
z^{T} \nabla g_{i}\left(x^{*}\right)=0 & j \in J_{1} \equiv\left\{j: g_{j}\left(x^{*}\right)=0, \mu_{j}>0\right\}, \\
z^{T} \nabla g_{i}\left(x^{*}\right) \leq 0 & j \in J_{2} \equiv\left\{j: g_{j}\left(x^{*}\right)=0 \quad \mu_{j}=0\right\},
\end{array}
$$

it follows that

$$
z^{T} \nabla^{2} L\left(x^{*}, \lambda^{*}, \mu^{*}\right) z>0
$$

then, $x^{*}$ is a strict local minimum of problem (4.1).

\section{Generalized Reduced Gradient Algorithm}

The optimality conditions presented in the previous section provide the theoretical basis for the development of different optimization algorithms. Generalized Reduced Gradient (GRG) Algorithm is one of the most commonly used algorithms for solving constrained optimization.

As stated in the previous section, a constrained optimization problem can be expressed as:

$$
\begin{array}{ll}
\min & f(\boldsymbol{x}) \\
\text { s.t. } & \boldsymbol{h}(\boldsymbol{x})=0 \\
& \boldsymbol{g}(\boldsymbol{x}) \leq 0
\end{array}
$$


Among inequality constraints, they are subdivided into two categories: boundary constraints $\left(L_{i} \leq x_{i} \leq U_{i}\right.$, one of $L_{i}, U_{i}$ could be infinite) and function inequality constraints. Boundary constraints are separated from other inequality constraints because they are treated differently in determining the step length in a search direction as we'll see later. Function inequality constraints can be transformed into equality constraints by adding slack variables. For example, an inequality constraint

$$
g_{j}(x) \leq 0
$$

By adding slack variable $x_{n+\jmath}$, it can be redefined as

$$
h_{m+j}(x)=g_{j}(x)+x_{n+j}=0
$$

Where $x_{n+j} \geq 0$. Contrary to slack variables, variable $\mathrm{x}_{1}, \mathrm{x}_{2}, \ldots, \mathrm{x}_{\mathbf{n}}$ are called natural variables. Therefore, an inequality constraint, which is the function of natural variables, is transformed into an equality constraint, which is the function of natural variables plus a slack variable, and a boundary constraint on that slack variable.

By treating boundary constraints separately and transforming inequality constraints to equality constraints, problem (4.1) is converted to the following equivalent form:

$$
\begin{array}{lll}
\min & f(\boldsymbol{x}) & \boldsymbol{x}=\left[x_{1}, x_{2}, \cdots, x_{\mathrm{n}}\right]^{\mathrm{T}} \\
\text { s.t. } & h_{j}(\boldsymbol{x})=0 & j=1, \cdots, m \\
& L_{i} \leq x_{i} \leq U_{i} & i=1, \cdots, n
\end{array}
$$


However, keep in mind that $\mathrm{m}, \mathrm{n}$ are different numbers here with those in formulation (4.1) and $\boldsymbol{x}$ is the set of natural variables and slack variables. (4.6) is the formulation we are going to use for describing GRG algorithm.

The generalized reduced gradient algorithm is based on a very basic optimization procedure which transforms a constrained optimization problem into one that is unconstrained or bound-constrained. It classes the variables as independent and dependent, and substitutes into the objective function the expressions obtained from the equality constraints in independent variables for the dependent variables. This essentially reduces the original problem to an unconstrained or bound-constrained problem of reduced dimension (Lasdon, et al., 1978).

In terms of dependent and independent variables, the constraints in formulation (3.6) can be written as

$$
\boldsymbol{h}(\boldsymbol{x})=\boldsymbol{h}\left(\boldsymbol{x}_{\mathrm{I}}, \boldsymbol{x}_{\mathrm{D}}\right)=0
$$

Where $\boldsymbol{x}_{\mathrm{I}}$ are the set of independent variables, $\boldsymbol{x}_{\mathrm{D}}$ are the set of dependent variables. $\boldsymbol{x}_{\mathrm{D}}$ are solved such that

$$
x_{\mathrm{D}}=\varphi\left(x_{\mathrm{I}}\right)
$$

According to the formulation (4.6), the number of $\boldsymbol{x}_{\mathbf{I}}$ is $(\mathrm{n}-\mathrm{m})$ and $\boldsymbol{x}_{\mathbf{D}}$ is $\mathrm{m}$. Thus the objective function can be written as

$$
f(x)=f\left(x_{\mathbf{I}}, x_{\mathbf{D}}\right)=f\left(x_{\mathbf{I}}, \varphi\left(x_{\mathbf{I}}\right)\right)=\mathrm{F}\left(x_{\mathbf{I}}\right)
$$

The original problem is now transformed into a simpler reduced problem of (n-m) dimension:

$\min F(x)$

$$
\text { s.t. } \quad L \leq x \leq U
$$


Where $\boldsymbol{L}$ and $\boldsymbol{U}$ are the bound vectors for $\boldsymbol{x}$. The function $\mathrm{F}(\boldsymbol{x})$ is called the reduced objective.

The concept of the reduced gradient is best described by the following simple example (Edgar and Himmelblau, 1988):

$$
\begin{array}{ll}
\min & f\left(x_{1}, x_{2}\right) \\
\text { s.t. } & h\left(x_{1}, x_{2}\right)=0
\end{array}
$$

The total derivatives of each function are:

$$
\begin{aligned}
& d f(\boldsymbol{x})=\frac{\partial f(\boldsymbol{x})}{\partial x_{1}} d x_{1}+\frac{\partial f(\boldsymbol{x})}{\partial x_{2}} d x_{2} \\
& d h(\boldsymbol{x})=\frac{\partial h(\boldsymbol{x})}{\partial x_{1}} d x_{1}+\frac{\partial h(\boldsymbol{x})}{\partial x_{2}} d x_{2}=0
\end{aligned}
$$

If $x_{1}$ is designated to be the dependent variable, $x_{2}$ to be the independent variable, then $\mathrm{d} x_{1}$ can be eliminated from Eq. (4.9) by use of Eq. (4.10) since

$$
d x_{1}=-\frac{\left[\partial h(x) / \partial x_{2}\right]}{\left[\partial h(x) / \partial x_{1}\right]} d x_{2}
$$

Therefore,

$$
d f(\boldsymbol{x})=\left\{\left[\frac{\partial f(\boldsymbol{x})}{\partial x_{2}}\right]-\left[\frac{\partial f(\boldsymbol{x})}{\partial x_{1}}\right]\left[\frac{\partial h(\boldsymbol{x})}{\partial x_{1}}\right]^{-1}\left[\frac{\partial h(\boldsymbol{x})}{\partial x_{2}}\right]\right\} d x_{2}
$$


The expression in the brace is called the reduced gradient. Of course, in this problem the reduced gradient contains only one element because there is only one independent variable. The general form of the reduced gradient is:

$$
g_{R}^{T}=\left[\frac{\partial f(\boldsymbol{x})}{\partial \boldsymbol{x}_{\mathrm{r}}}\right]^{T}-\left[\frac{\partial f(\boldsymbol{x})}{\partial \boldsymbol{x}_{\mathrm{D}}}\right]^{T}\left[\frac{\partial \boldsymbol{h}(\boldsymbol{x})}{\partial \boldsymbol{x}_{\mathrm{D}}}\right]^{-1}\left[\frac{\partial \boldsymbol{h}(\boldsymbol{x})}{\partial \boldsymbol{x}_{\mathrm{I}}}\right]
$$

The generalized reduced-gradient algorithm can be stated in five basic steps (Edgar and Himmelblau, 1988). In the text, $\boldsymbol{x}_{\mathbf{I}}$ and $\boldsymbol{x}_{\mathrm{D}}$ bear superscripts $\mathrm{k}$ since their components may well change from stage to stage. Also to simplify the notation, we denote $f\left(\boldsymbol{x}^{\mathrm{k}}\right)$ as $f^{\mathrm{k}}$ and $\boldsymbol{h}\left(\boldsymbol{x}^{\mathrm{k}}\right)$ as $\boldsymbol{h}^{\mathrm{k}}$.

\section{Step 1 Determine the search components for the independent variables.}

At stage $\mathrm{k}$, linearize the constraints at the feasible point $x^{\mathrm{k}}$ and compute the reduced gradient:

$$
\left(g_{R}{ }^{k}\right)^{T}=\left[\frac{\partial f^{k}}{\partial \boldsymbol{x}_{\mathrm{I}}^{k}}\right]^{T}-\left[\frac{\partial f^{k}}{\partial \boldsymbol{x}_{\mathrm{D}}^{k}}\right]^{T}\left[\frac{\partial \boldsymbol{h}^{k}}{\partial \boldsymbol{x}_{\mathrm{D}}^{k}}\right]^{-1}\left[\frac{\partial \boldsymbol{h}^{k}}{\partial \boldsymbol{x}_{\mathrm{I}}^{k}}\right]
$$

Then the search direction components of the independent variables are established as:

$$
\Delta_{i}^{k}=-g_{R i}^{k}
$$

However, $\Delta_{i}^{k}$ is selected as 0 if $x_{i}$ is at one of its bounds and the step would exceed the bound. That is,

$$
\begin{aligned}
& \Delta_{i}^{k}=0 \quad \text { if } x_{i}^{k}=U_{\mathrm{i}} \quad g_{\mathrm{Ri}}<0 \\
& =L_{\mathrm{i}} \quad g_{\mathrm{Ri}}>0
\end{aligned}
$$




\section{Step 2 Determine the search components for the dependent variables.}

A desired search direction would be along the surface of the constraints. This is accomplished by finding the tangent to $\boldsymbol{h}(\boldsymbol{x})=0$ at the point $\left(\boldsymbol{x}_{\mathrm{I}}^{k}, \boldsymbol{x}_{\mathrm{D}}^{k}\right)$. That is,

$$
\begin{aligned}
& d \boldsymbol{h}=\frac{\partial \boldsymbol{h}}{\partial \boldsymbol{x}_{\mathrm{I}}} d \boldsymbol{x}_{\mathrm{I}}+\frac{\partial \boldsymbol{h}}{\partial \boldsymbol{x}_{\mathrm{D}}} d \boldsymbol{x}_{\mathrm{D}}=0 \\
& d \boldsymbol{x}_{\mathrm{D}}=-\left[\frac{\partial \boldsymbol{h}}{\partial \boldsymbol{x}_{\mathrm{D}}}\right]^{-1}\left[\frac{\partial \boldsymbol{h}}{\partial \boldsymbol{x}_{\mathrm{I}}}\right] d \boldsymbol{x}_{\mathrm{I}}
\end{aligned}
$$

Therefore, the search direction components of the dependent variables are:

$$
\Delta_{\mathbf{D}}^{k}=-\left[\frac{\partial \boldsymbol{h}^{k}}{\partial \boldsymbol{x}_{\mathbf{D}}^{k}}\right]^{-1}\left[\frac{\partial \boldsymbol{h}^{k}}{\partial \boldsymbol{x}_{\mathbf{I}}^{k}}\right] \Delta_{\mathrm{I}}^{k}
$$

\section{Step 3 Improving the value of the objective function.}

Minimize $f\left(\mathbf{x}_{\mathbf{I}}^{k}+\lambda \Delta_{\mathbf{I}}^{k}, \mathbf{x}_{\mathbf{D}}^{k}+\lambda \Delta_{\mathbf{D}}^{k}\right)$ with respect to $\lambda(\lambda>0)$ by a one-dimensional search. The new point is:

$$
\begin{aligned}
& \boldsymbol{x}_{\mathrm{I}}^{k+1}=\boldsymbol{x}_{\mathrm{I}}^{k}+\lambda^{k} \Delta_{\mathrm{I}}^{k} \\
& \widetilde{\boldsymbol{x}}_{\mathbf{D}}^{k+1}=\boldsymbol{x}_{\mathbf{D}}^{k}+\lambda^{k} \Delta_{\mathbf{D}}^{k} \quad \widetilde{\mathbf{x}}_{\mathbf{D}} \text { is a tentative point }
\end{aligned}
$$




\section{Step 4 Use Newton's method to regain feasibility of dependent variables.}

In general, some of the components of $\widetilde{\mathbf{x}}_{\mathbf{D}}^{k+1}$ are not feasible, hence $\boldsymbol{h}\left(\boldsymbol{x}_{I}^{k+1}, \widetilde{\boldsymbol{x}}_{D}^{k+1}\right) \neq 0$. In order to return from a point away from constraints back to a point satisfying the constraints, $\widetilde{\boldsymbol{x}}_{\mathrm{D}}^{k+1}$ is modified by Newton's method:

$$
\boldsymbol{x}_{\mathrm{D}}^{k+1}=\widetilde{\boldsymbol{x}}_{\mathrm{D}}^{k+1}-\left[\frac{\partial \boldsymbol{h}\left(\boldsymbol{x}_{\mathrm{I}}^{k+1}, \widetilde{\boldsymbol{x}}_{\mathrm{D}}^{k+1}\right)}{\partial \boldsymbol{x}_{\mathrm{D}}^{k}}\right]^{-1} \boldsymbol{h}\left(\boldsymbol{x}_{\mathrm{I}}^{k+1}, \widetilde{\boldsymbol{x}}_{\mathrm{D}}^{k+1}\right)
$$

Several iterations of Newton's method may be needed to find a feasible point. Figure 4.1 illustrates the move from $\boldsymbol{x}^{k}$ to $\left(\boldsymbol{x}_{\mathrm{I}}^{k+1}, \widetilde{x}_{\mathbf{D}}^{k+1}\right)$ and then to $\left(x_{\mathrm{I}}^{k+1}, x_{\mathbf{D}}^{k+1}\right)$ by Newton's method.

Note: Newton's method finds the desired root $\boldsymbol{x}_{\mathrm{D}}^{k+1}$ with $\widetilde{\boldsymbol{x}}_{\mathbf{D}}^{\mathbf{k}+\mathbf{1}}$ as the starting point. By expanding $\boldsymbol{h}\left(\boldsymbol{x}_{\mathbf{I}}^{k+1}, \boldsymbol{x}_{\mathrm{D}}^{k+1}\right)$ and equating it to zero, we get

$$
\boldsymbol{h}\left(\boldsymbol{x}_{\mathbf{I}}^{k+1}, \boldsymbol{x}_{\mathbf{D}}^{k+1}\right) \approx \boldsymbol{h}\left(\boldsymbol{x}_{\mathbf{I}}^{k+1}, \tilde{\boldsymbol{x}}_{\mathbf{D}}^{k+1}\right)+\frac{\partial \boldsymbol{h}\left(\boldsymbol{x}_{\mathbf{I}}^{k+1}, \tilde{\boldsymbol{x}}_{\mathbf{D}}^{k+1}\right)}{\partial \boldsymbol{x}_{\mathbf{D}}^{k}}\left(\boldsymbol{x}_{\mathbf{D}}^{k+1}-\widetilde{\boldsymbol{x}}_{\mathbf{D}}^{k+1}\right)=0
$$

\section{Step 5 Procedure on lack of convergence of Newton's method.}

(a) If $x^{k+1}$ is a feasible point and $f\left(x^{k+1}\right)<f\left(x^{k}\right)$, adopt $x^{k+1}$, and start with step 1 again.

(b) If $\boldsymbol{x}^{k+1}$ is a feasible point and $f\left(x^{k+1}\right)>f\left(x^{k}\right)$, reduce $\lambda$ by a factor and start step 4 over again.

(c) If Newton's method fails to find a feasible point after certain times, reduce $\lambda$ by a factor and start step 4 over again. 


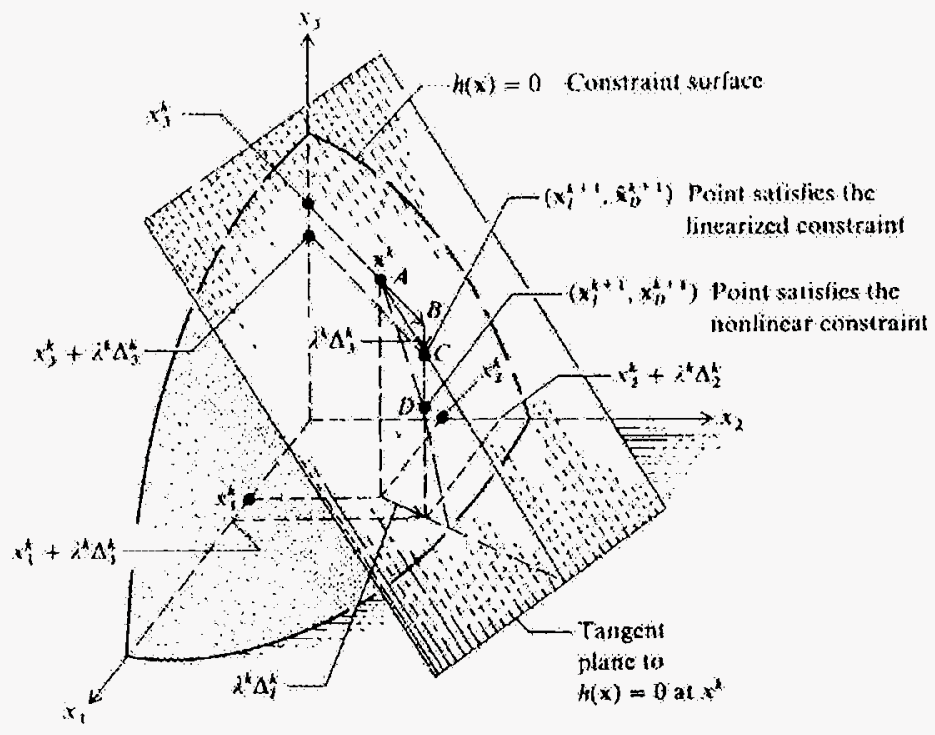

Figure 4.1 Illustration Of Using Newton's Method To Regain Feasibility

(d) If neither (a), (b) nor (c) occur, or (b) and (c) are unsuccessful after several passes, change the basis by exchanging a dependent variable with a former independent variable. This process is termed a change of basis.

Theoretically, the stopping condition for optimization is when KKT conditions are satisfied. However, in practice, we can use a simpler condition:

$$
\left|\frac{f\left(x^{k+1}\right)-f\left(x^{k}\right)}{f\left(x^{k}\right)}\right|<\varepsilon
$$

Where $\varepsilon$ is a small number.

A numeric example is given as following to illustrate the GRG algorithm. 


\section{EXAMPLE:}

$$
\begin{array}{ll}
\max & f(\boldsymbol{x})=\left(2 x_{1}-0.5 x_{1}^{2}\right)+\left(3 x_{2}-0.5 x_{2}^{2}\right) \\
\text { s.t. } & h(\mathbf{x})=x_{1}^{2}+x_{2}^{2}+x_{3}-1=0 \\
& -2 \leq x_{j} \leq 2 \quad j=1,2,3
\end{array}
$$

Solution: We minimize $-f(\boldsymbol{x})$. In the text below, $f(\boldsymbol{x})$ actually refers to $-f(\boldsymbol{x})$.

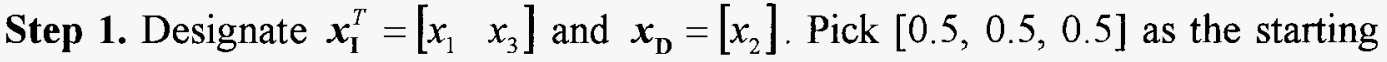
point. The reduced gradient is:

$$
g_{R}^{T}=\left[\frac{\partial f(\boldsymbol{x})}{\partial \boldsymbol{x}_{\mathbf{I}}}\right]^{T}-\left[\frac{\partial f(\boldsymbol{x})}{\partial \boldsymbol{x}_{\mathbf{D}}}\right]^{T}\left[\frac{\partial \boldsymbol{h}(\boldsymbol{x})}{\partial \boldsymbol{x}_{\mathbf{D}}}\right]^{-1}\left[\frac{\partial \boldsymbol{h}(\boldsymbol{x})}{\partial \boldsymbol{x}_{\mathbf{I}}}\right]
$$

where

$$
\begin{array}{cc}
\frac{\partial f(\boldsymbol{x})}{\partial \boldsymbol{x}_{\mathbf{I}}}=-\left[\begin{array}{c}
2-x_{1} \\
0
\end{array}\right] & \frac{\partial f(\boldsymbol{x})}{\partial \boldsymbol{x}_{\mathrm{D}}}=-\left(3-x_{2}\right) \\
\frac{\partial \boldsymbol{h}(\boldsymbol{x})}{\partial \boldsymbol{x}_{\mathrm{I}}}=\left[\begin{array}{ll}
2 x_{1} & 1
\end{array}\right] & \frac{\partial \boldsymbol{h}(\boldsymbol{x})}{\partial \boldsymbol{x}_{\mathrm{D}}}=2 x_{2} \\
\left(g_{R}^{0}\right)^{T}=-\left[\begin{array}{ll}
2-x_{1}^{0} & 0
\end{array}\right]-\left[-\left(3-x_{2}^{0}\right)\right]\left[\frac{1}{2 x_{2}^{0}}\right]\left[\begin{array}{ll}
2 x_{1}^{0} & 1
\end{array}\right]=\left[\begin{array}{ll}
1 & 2.5
\end{array}\right]
\end{array}
$$

As the result, the search direction of the independent variable is,

$$
\Delta_{\mathrm{I}}^{0}=-g_{R}^{0}=\left[\begin{array}{c}
-1 \\
-2.5
\end{array}\right]
$$

Step 2. The search direction of the dependent variable is:

$$
\Delta_{\mathrm{D}}^{0}=-\left[\frac{\partial \boldsymbol{h}^{0}}{\partial \boldsymbol{x}_{\mathrm{D}}^{0}}\right]^{-1}\left[\frac{\partial \boldsymbol{h}^{0}}{\partial \boldsymbol{x}_{\mathbf{I}}^{0}}\right] \Delta_{\mathrm{I}}^{0}=-\left[\frac{1}{2 x_{2}^{0}}\right]\left[\begin{array}{ll}
2 x_{1}^{0} & 1
\end{array}\right] \Delta_{\mathbf{I}}^{0}=3.5
$$

Step 3. From above, we get 


$$
\begin{aligned}
& \Delta^{0}=\left[\begin{array}{c}
-1 \\
3.5 \\
-2.5
\end{array}\right] \\
& f\left(x^{0}+\lambda \Delta^{0}\right)=-\left\{\left[2(0.5-\lambda)-0.5(0.5-\lambda)^{2}\right]+\left[3(0.5+3.5 \lambda)-0.5(0.5+3.5 \lambda)^{2}\right]\right\}
\end{aligned}
$$

Minimize $f\left(x^{0}+\lambda \Delta^{0}\right)$ with respect to $\lambda$ by setting $(\partial f / \partial \lambda)=0$ and solving the resulting equation. We get

$$
\begin{aligned}
& \lambda^{0}=0.547 \\
& \boldsymbol{x}_{\mathbf{I}}^{1}=\left[\begin{array}{l}
0.5 \\
0.5
\end{array}\right]+0.547\left[\begin{array}{c}
-1 \\
-2.5
\end{array}\right]=\left[\begin{array}{l}
-0.047 \\
-0.867
\end{array}\right] \\
& \widetilde{\boldsymbol{x}}_{\mathbf{D}}^{1}=[0.5]+0.547[3.5]=2.415
\end{aligned}
$$

Step 4. Check if $h(x)=0$,

$$
h^{1}=(-0.047)^{2}+(2.415)^{2}+(-0.867)-1=3.967 \neq 0
$$

Constraint is not satisfied. Applying Newton's method to modify $\widetilde{x}_{2}^{1}$ :

$$
\begin{aligned}
& \frac{\partial h^{1}}{\partial x_{2}^{1}}=2 \widetilde{x}_{2}^{1}=4.829 \\
& \hat{x}_{2}^{1}=2.415-\left(\frac{1}{4.829}\right)(3.967)=1.594
\end{aligned}
$$

Successively applying Newton's method, starting each time with the previously found $\hat{x}_{2}^{1}$, until a feasible $x_{2}^{1}$ is found. We give the result here:

$$
x_{2}^{1}=1.366
$$

\section{Step 5.}

$$
\begin{aligned}
& f\left(x^{0}\right)=-2.25 \\
& f\left(x^{1}\right)=-3.07
\end{aligned}
$$


$\therefore \quad f\left(x^{1}\right)<f\left(x^{0}\right)$

$x^{1}$ is a better point and adopted as new starting point. New search starts with step 1 again.

Normally, the starting point, $x^{0}$, is a feasible solution. But that's not necessary. If the starting point, $x^{0}$, is not feasible, the optimization is done in two phases. In phases $\mathrm{I}$, $x^{0}$ is made feasible by adding artificial variables to satisfy the equality constraints, then forcing them to zero values thus finding a new feasible point (Hwang, et al., 1972). Let an artificial variable, $x_{\mathrm{n}+\mathrm{i}}$, satisfy the condition,

$$
0 \leq x_{n+i} \leq \infty
$$

If, at the starting point $x^{0}$, the $i$ th constraint violates the equality condition by being greater than zero, i.e., $h_{\mathrm{i}}\left(\boldsymbol{x}^{0}\right)>0$, it is made feasible by subtracting the artificial variable, $x_{\mathrm{n}+\mathrm{i}}$

$$
h_{\mathrm{i}}\left(\boldsymbol{x}^{0}\right)-x_{\mathrm{n}+\mathrm{i}}=0
$$

Likely, if $h_{i}\left(x^{0}\right)<0$, it is made feasible by adding the artificial variable,

$$
h_{\mathrm{i}}\left(\boldsymbol{x}^{0}\right)+x_{\mathrm{n}+\mathrm{i}}=0
$$

The objective function to be minimized in phase I becomes the sum of all artificial variables,

$$
\min \sum_{i \in N E} x_{n+i} \quad N E=\left\{i \mid h_{i}(x) \neq 0\right\}
$$

The artificial variables are forced to zero by minimization. When all of the artificial variables are zero, the solution, $\boldsymbol{x}$, becomes feasible. The second phase optimization begins with this new feasible solution. 


\section{Description of the Problem}

\section{Variable Temperature Heat Source}

For a given heat source fluid and a fixed ambient temperature $\mathrm{T}_{0}$, the maximum useful work (per unit weight of heat source fluid) is a function of only $T_{h s}^{i n}$, the heat source temperature:

$$
w_{\max }=\left(h_{h s}^{\text {in }}-h_{0}\right)-T_{0}\left(s_{h s}^{i n}-s_{0}\right)
$$

Where $h_{h s}^{\text {in }}$ is the inlet enthalpy of the heat source fluid;

$s_{h s}^{\text {in }}$ is the inlet entropy of the heat source fluid;

$h_{0}$ is the enthalpy of the heat source fluid at ambient temperature;

$s_{0}$ is the entropy of the heat source fluid at ambient temperature.

A plot of this value is given in Figure 4.2. The heat source fluid in Figure 4.2 is saturated water when its temperature is above $100^{\circ} \mathrm{C}$ and subcooled water at 1 atm when the temperature is below $100^{\circ} \mathrm{C}$.

Any real process will have nonreversible steps that will result in a net work output less than maximum useful work. These irreversibilities can be evaluated for each step of the conversion process. For example, any heat exchange step destroys availability due to the finite temperature difference between the fluids. Although big temperature difference favors the heat transfer process, thus requiring less heat transfer surface area, it will cause a degradation of availability and a corresponding loss in thermodynamic performance of the process. Optimal operation with the minimum irreversibility occurs when the heat capacities of both streams are constant. This situation produces a balanced exchanger in 


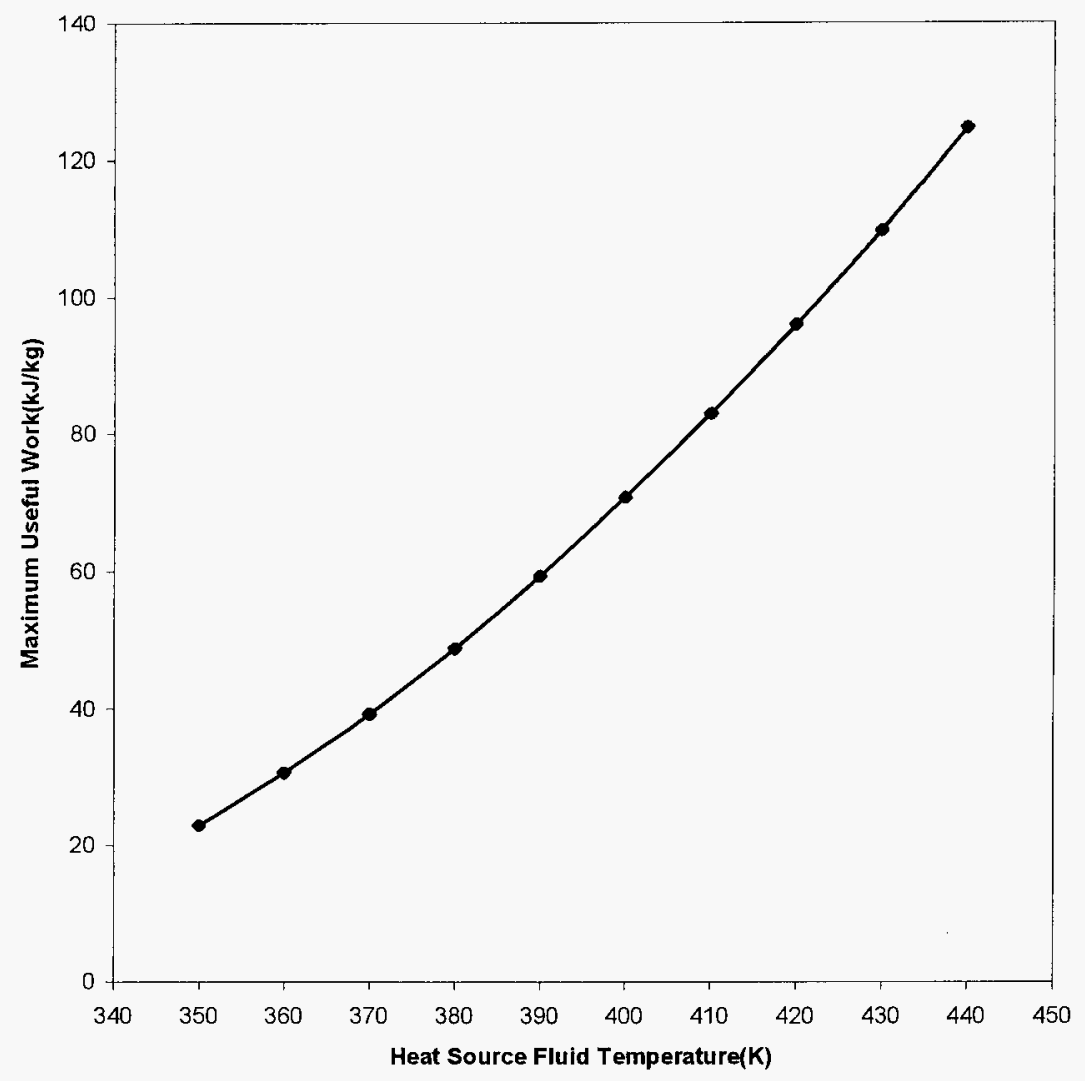

Figure 4.2 Maximum Useful Work Plotted as a Fuction of Heat Source Fluid Temperature

which the temperature difference $\Delta \mathrm{T}$ between streams can be kept uniform. In the heat exchanger, a "pinch" or minimum temperature difference occurs at the point where the liquid begins to evaporate. Efficient resource utilization will result when (Milora, 1976):

1. Most of the heat is extracted from the heat source fluid before disposal.

2. Temperature differentials across heat transfer surfaces are maintained at minimum practical levels.

3. Turbines and feed pumps are carefully designed for optimum efficiency.

4. Heat is rejected from the thermodynamic cycle at a temperature near the minimum ambient temperature. 
Quantitatively, one can define a cycle efficiency factor that is a measure of the fraction of the available energy of the heat source fluid that is converted into useful work. Second law thermal efficiency $\eta_{2}$, is defined in terms of net power output $W_{\text {net, }}$, the heat source flow rate, $m_{h s}$, and the availability:

$$
\eta_{2}=\frac{W_{n e t}}{m_{h s}\left[\left(h_{h s}^{i n}-h_{0}\right)-T_{0}\left(s_{h s}^{i n}-s_{0}\right)\right]}
$$

$\eta_{2}$ is a direct measure of the efficiency of resource utilization, because for a fixed $T_{h s}^{i n}$, higher value of $\eta_{2}$ corresponds to lower flow rate of the source fluid for a given net power output. $\eta_{2}$ should not be confused with the first law thermal efficiency, $\eta_{1}$, which is a measure of how efficiently the transferred heat is converted into work.

$$
\eta_{1}=\frac{W_{n e t}}{m_{h s}\left(h_{h s}^{\text {in }}-h_{h s}^{\text {out }}\right)}
$$

Where $h_{h s}^{\text {out }}$ is the outlet enthalpy of the heat source fluid. The quality of the heat source and the availability of the disposed heat source fluid at temperatures above ambient are not considered in calculating $\eta_{1}$.

\section{Optimization Model for the Cycle}

Optimization is a very powerful tool in the engineering decision-making. A typical engineering problem can be expressed as follows: We have a process that can be represented by some equations and/or experimental data. We also have a performance criterion in mind, for example, to achieve maximum thermal efficiency. The goal of the 
optimization is to find an operating condition, which is represented by the values of the some variables, that yields the best performance.

Problem formulation is perhaps the most crucial step in resolving a problem that involves optimization. Problem formulation requires identifying a conceptual statement of a given application, and organizing them into a standard mathematical form. Namely

1. The objective function

\section{Constraints}

The objective function represents, in terms of the key variables, the process being analyzed. The constraints describe the interrelationships of the key variables. Constraints in optimization problems arise from physical bounds on the variables, empirical relations, physical laws, etc.

For this ammonia-based combined power/cooling cycle, our optimization objective is to find the maximum thermal efficiency. By looking into the cycle (Fig. 2.7), we can see that the high and low pressure of the cycle can be freely changed and have to be chosen by the designer. In addition, the temperatures of the absorber, boiler, rectifier and superheater, are also free variables.

From the previous parametric analysis, we have concluded higher concentration of the ammonia strong solution always results in better cycle performance. Therefore, a saturation state is desired in the absorber. For saturated ammonia solution, when its temperature and pressure are fixed, its concentration is determined. No additional variable is added into the set of the free variables.

In the boiler, the leaving ammonia weak solution is bound to be saturated and the leaving ammonia vapor is in an equilibrium state with the weak solution. So when the 
pressure and temperature of the boiler, and the concentration of the feeding ammonia strong solution are fixed, the concentration of the weak solution and ammonia vapor along with their fractions are determined.

For heat source, there are three unknown variables: $m_{h s}, T_{h s}^{\text {in }}, T_{h s}^{\text {out }}$. However, due to the energy balance in the boiler and superheater, the energy transferred out of the heat source equals the energy transferred into the cycle. So only two variables are actually unknown. Any two of them can be chosen as free variables. We choose $T_{h s}^{i n}, T_{h s}^{\text {out }}$.

In summary, this ammonia-based combined power/cooling cycle has eight free

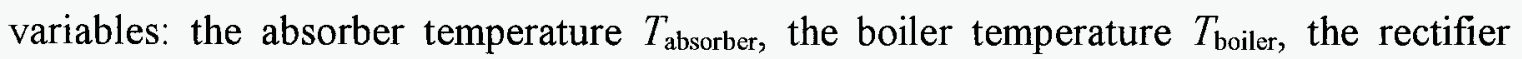

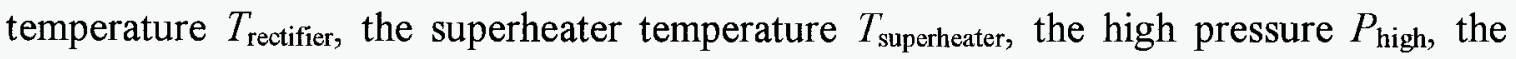
low pressure $P_{\text {low, }}$ the heat source entrance temperature $T_{h s}^{i n}$, and the heat source exit temperature $T_{h s}^{\text {out }}$. The cycle performance depends on the selection of the values for these eight free variables. Each combination of the eight values represents a distinctive operating condition of the cycle. Searching for optimum values for these variables is the task of this optimization work. Consequently, the objective function of the optimization of the cycle can be written as:

$$
\eta=f\left(T_{\text {absorber }}, T_{\text {boiler, }}, T_{\text {rectifier, }}, T_{\text {superheater, }}, P_{\text {high }}, P_{\text {low }}, T_{h s}^{\text {in }}, T_{h s}^{\text {out }}\right)
$$

As we have discussed above, second law thermal efficiency $\eta_{2}$ is the true reflection of the efficiency of resource utilization, it is chosen as our optimization objective.

After we have decided on the objective function, the next step is to find the constraints, which are required for the practicability of the cycle. These constraints define feasible region. By common sense, the temperature of the rectifier has to be lower than 
the temperatures of the boiler and superheater. Besides, the temperature of the boiler has to be greater than a minimum value, which is the bubble point of the feeding ammonia solution. Otherwise, no ammonia will be boiled off from the ammonia solution. Similarly, the temperature of the rectifier has to be greater than a minimum value so ammonia vapor entering the rectifier unit won't be totally condensed.

For the turbine, lowering its exhaust pressure will increase the thermal efficiency. However, the moisture content of the fluid leaving the turbine is increased at the same time. This increased moisture content decreases the efficiency of an actual turbine. In addition, the impingement of liquid droplet on the turbine blades leads to a serious erosion problem. In practice, it is desirable to keep the moisture content less than about 10 percent at the low pressure end of the turbine (Wark, 1988). Therefore, the quality of the ammonia vapor at the exit of the turbine has to be greater than $90 \%$.

In the cycle (shown in Figure 2.7), after being pumped to the high pressure, ammonia strong solution is separated into two streams (point 2). By common sense, their fractions are between 0 and 1 . Of course, when either of them is between 0 and 1 , the other must be. So only one constraint is added into the set of the constraints. The fraction of the solution at point 2 separated into the stream at point $2 ", f_{2}$ ", is randomly picked. In addition, the flow rate of the ammonia vapor leaving the boiler has to be less than that of feeding ammonia solution. Therefore, the fraction flowing through point $4, f_{4}$, which is defined by $m_{4} / m_{1}$, is between 0 and 1 .

Finally, the most common type of constraints in the thermodynamic cycle is the temperature difference limit on the heat exchangers. They are expressed mathematically with other constrains discussed as follows: 


$$
\begin{array}{ll}
\text { - } & T_{\text {boiler }} \geq T_{\text {rectifier }} \\
\text { - } & T_{\text {superheater }} \geq T_{\text {rectifier }} \\
\text { - } & T_{\text {boiler }} \geq T_{\text {boilermin }} \\
\text { - } & T_{\text {rectifier }} \geq T_{\text {rectifiermin }} \\
\text { - } & x_{\text {turbine }} \geq 0.9 \\
\text { - } & 0 \leq f_{4} \leq 1 \\
\text { - } & T_{10}-T_{2}, \geq \Delta T_{\min } \\
\text { - } & T_{\mathrm{I}}-T_{\text {superheater }} \geq \Delta T_{\min } \\
\text { - } & T_{\mathrm{III}}-T_{3} \geq \Delta T_{\min } \\
\text { - } & T_{\mathrm{II}}-T_{\text {boiler }} \geq \Delta T_{\min } \\
& \Delta T_{\text {pin }} \geq \Delta T_{p i n}^{\min } \\
\text { - } & \\
& \\
&
\end{array}
$$

Where

$x_{\text {turbine }}$ is the quality of the vapor at turbine exit;

$T_{\mathrm{x}}$ : temperature at point $\mathrm{x}$ (refer to Fig. 2.7 );

$\Delta T_{\min }$ : minimum temperature difference required in the heat exchangers;

$\Delta T_{\text {pin }}:$ temperature difference at pinch point in the boiler;

$\Delta T_{p i n}^{\min }:$ minimum temperature difference required at pinch point.

The variables that appear in the constraints are free variables or the functions of free variables. Now, we have got an objective function and a complete set of constraints. The 
formulation of the optimization model for the ammonia-base combined power/cooling cycle is finished. Optimization work is ready to start.

\section{Optimization Program}

For optimization, we use the GRG2 program written by Dr. Lasdon. GRG2 is by far the most widely distributed nonlinear programming code (More, J. J., Wright, S. J., 1993). It uses an implementation of the generalized reduced gradient (GRG) algorithm. It has both Fortran and $\mathrm{C}$ version. $\mathrm{C}$ version is used in the current research. In the program, the user is required to provide a subroutine gcomp. The values of the objective function and constraints are calculated in this subroutine and provided to the main optimization program. The user also provides data specifying the upper and lower bounds on the free variables and initial values of the free variables. In the subroutine gcomp, the thermodynamic calculation of the ammonia-based combined power/cooling cycle is done to get the objective function, which is thermal efficiency, and constraints. Property programs are called to calculate the properties of ammonia/water mixture at various state points.

\section{Optimization Results}

In this section, optimization results are presented. Two typical heat source temperatures are studied. One is $440 \mathrm{~K}$, the other is $360 \mathrm{~K}$. The ambient temperatures for both cases are assumed as $290 \mathrm{~K}$. Their optimum working conditions are presented. 


\section{Resource Temperature of $440 \mathrm{~K}$}

This heat source temperature is within the range of geothermal source and a CPC solar collector. The cycle is optimized in terms of second law thermal efficiency. The optimum working conditions are presented in Table 4.1:

Table 4.1 Optimum Working Conditions

\begin{tabular}{|c|c|c|c|c|c|c|}
\hline Point & $\mathrm{T}(\mathrm{K})$ & $\mathrm{P}(\mathrm{bar})$ & $\mathrm{h}(\mathrm{kJ} / \mathrm{kg})$ & $\mathrm{s}(\mathrm{kJ} / \mathrm{kg} . \mathrm{K})$ & $\mathrm{X}$ & $\begin{array}{c}\text { Flow Rate } \\
(\mathrm{kg} / \mathrm{s})\end{array}$ \\
\hline 1 & 295.0 & 8.6 & 66.0 & 0.3712 & 0.9500 & 1.0000 \\
\hline 2 & 296.1 & 52.0 & 72.9 & 0.3712 & 0.9500 & 1.0000 \\
\hline 3 & 313.4 & 52.0 & 156.2 & 0.6447 & 0.9500 & 1.0000 \\
\hline 4 & 374.3 & 52.0 & 1315.6 & 3.8000 & 0.9943 & 0.7720 \\
\hline 5 & 374.3 & 52.0 & 358.2 & 1.4403 & 0.7999 & 0.0000 \\
\hline 6 & 374.3 & 52.0 & 1315.6 & 3.8000 & 0.9943 & 0.7720 \\
\hline 7 & 435.0 & 52.0 & 1547.1 & 4.3606 & 0.9943 & 0.7720 \\
\hline 8 & 300.5 & 8.6 & 1270.9 & 4.3606 & 0.9943 & 0.7720 \\
\hline 9 & 300.5 & 8.6 & 1270.9 & 4.3606 & 0.9943 & 0.7720 \\
\hline 10 & 374.3 & 52.0 & 358.2 & 1.4403 & 0.7999 & 0.2280 \\
\hline 11 & 301.1 & 52.0 & -7.3 & 0.3568 & 0.7999 & 0.2280 \\
\hline 12 & 301.2 & 8.6 & -7.3 & 0.3775 & 0.7999 & 0.2280 \\
\hline
\end{tabular}

Boiler Heat Input:

Superheat Input:

Absorber Heat Rejection:

Turbine Work Output:

Vapor Quality at Turbine Exit:

Pump Work Input:

Total Heat Input:

Total Work Output:

First Law Efficiency:

Heat Source Flow Rate:

Heat Source Entrance Temperature:

Heat Source Exit Temperature:

Second law efficiency:
$941.1 \mathrm{~kJ} / \mathrm{s}$

$178.7 \mathrm{~kJ} / \mathrm{s}$

$913.5 \mathrm{~kJ} / \mathrm{s}$

$213.3 \mathrm{~kW}$

$97.55 \%$

$6.9 \mathrm{~kW}$

$1119.8 \mathrm{~kJ} / \mathrm{s}$

$206.37 \mathrm{~kW}$

$18.43 \%$

$2.783 \mathrm{~kg} / \mathrm{s}$

$440 \mathrm{~K}$

$345.4 \mathrm{~K}$

$59.63 \%$ 
It is noticed that the optimum concentration for ammonia strong solution is at 0.95. Though unnecessary, since the optimization program searches the entire concentration range to find the optimum concentration, another optimization is done at a fixed 0.9 concentration for ammonia strong solution to verify the previous optimization result. The result is given in Table 4.2 and shows that it has a lower second law efficiency.

Table 4.2 Optimum Working Conditions at $\mathrm{X}=0.9$

\begin{tabular}{|c|c|c|c|c|c|c|}
\hline Point & $\mathrm{T}(\mathrm{K})$ & $\mathrm{P}(\mathrm{bar})$ & $\mathrm{h}(\mathrm{kJ} / \mathrm{kg})$ & $\mathrm{s}(\mathrm{kJ} / \mathrm{kg} . \mathrm{K})$ & $\mathrm{X}$ & $\begin{array}{c}\text { Flow Rate } \\
(\mathrm{kg} / \mathrm{s})\end{array}$ \\
\hline 1 & 295.0 & 8.2 & 29.6 & 0.3438 & 0.9000 & 1.0000 \\
\hline 2 & 295.8 & 47.1 & 35.5 & 0.3438 & 0.9000 & 1.0000 \\
\hline 3 & 327.2 & 47.1 & 186.7 & 0.8293 & 0.9000 & 1.0000 \\
\hline 4 & 371.4 & 47.1 & 1329.4 & 3.8689 & 0.9939 & 0.5669 \\
\hline 5 & 371.4 & 47.1 & 326.4 & 1.3787 & 0.7771 & 0.0000 \\
\hline 6 & 371.4 & 47.1 & 1329.4 & 3.8689 & 0.9939 & 0.5669 \\
\hline 7 & 435.0 & 47.1 & 1559.5 & 4.4308 & 0.9939 & 0.5669 \\
\hline 8 & 302.3 & 8.2 & 1286.1 & 4.4308 & 0.9939 & 0.5669 \\
\hline 9 & 302.3 & 8.2 & 1286.1 & 4.4308 & 0.9939 & 0.5669 \\
\hline 10 & 371.4 & 47.1 & 326.4 & 1.3787 & 0.7771 & 0.4331 \\
\hline 11 & 300.8 & 47.1 & -22.6 & 0.3393 & 0.7771 & 0.4331 \\
\hline 12 & 301.0 & 8.2 & -22.6 & 0.3575 & 0.7771 & 0.4331 \\
\hline
\end{tabular}

Boiler Heat Input:

Superheat Input:

Absorber Heat Rejection:

Turbine Work Output:

Vapor Quality at Turbine Exit:

Pump Work Input:

Total Heat Input:

Total Work Output:

First Law Efficiency:

Heat Source Flow Rate:

Heat Source Entrance Temperature:

Heat Source Exit Temperature:

Second law efficiency:
$708.3 \mathrm{~kJ} / \mathrm{s}$

$130.4 \mathrm{~kJ} / \mathrm{s}$

$689.7 \mathrm{~kJ} / \mathrm{s}$

$155.0 \mathrm{~kW}$

$98.16 \%$

$5.9 \mathrm{~kW}$

$838.7 \mathrm{~kJ} / \mathrm{s}$

$149.07 \mathrm{~kW}$

$17.77 \%$

$2.072 \mathrm{~kg} / \mathrm{s}$

$440 \mathrm{~K}$

$344.8 \mathrm{~K}$

$57.86 \%$ 
At this heat source temperature, the optimum working conditions don't produce any cooling capacity. However, cooling capacity can be obtained at a non-optimum condition if necessary. Table 4.3 gives one of such conditions:

Table 4.3 A Working Condition with Cooling Capacity

\begin{tabular}{|c|c|c|c|c|c|c|}
\hline Point & $\mathrm{T}(\mathrm{K})$ & $\mathrm{P}(\mathrm{bar})$ & $\mathrm{h}(\mathrm{kJ} / \mathrm{kg})$ & $\mathrm{s}(\mathrm{kJ} / \mathrm{kg} . \mathrm{K})$ & $\mathrm{X}$ & $\begin{array}{c}\text { Flow Rate } \\
(\mathrm{kg} / \mathrm{s})\end{array}$ \\
\hline 1 & 295.0 & 4.6 & -125.9 & 0.1777 & 0.6131 & 1.0000 \\
\hline 2 & 295.2 & 31.8 & -122.4 & 0.1777 & 0.6131 & 1.0000 \\
\hline 3 & 374.8 & 31.8 & 334.2 & 1.5179 & 0.6131 & 1.0000 \\
\hline 4 & 403.0 & 31.8 & 1553.2 & 4.5993 & 0.9401 & 0.4078 \\
\hline 5 & 363.5 & 31.8 & 222.0 & 1.1859 & 0.6699 & 0.0648 \\
\hline 6 & 363.5 & 31.8 & 1377.5 & 4.1409 & 0.9911 & 0.3430 \\
\hline 7 & 366.4 & 31.8 & 1387.7 & 4.1684 & 0.9911 & 0.3430 \\
\hline 8 & 277.4 & 4.6 & 1139.2 & 4.1684 & 0.9911 & 0.3430 \\
\hline 9 & 285.0 & 4.6 & 1246.6 & 4.5514 & 0.9911 & 0.3430 \\
\hline 10 & 403.0 & 31.8 & 363.9 & 1.6145 & 0.4157 & 0.6570 \\
\hline 11 & 300.2 & 31.8 & -108.1 & 0.2668 & 0.4157 & 0.6570 \\
\hline 12 & 300.8 & 4.6 & -108.1 & 0.2774 & 0.4157 & 0.6570 \\
\hline
\end{tabular}

Boiler Heat Input:

Superheat Input:

Absorber Heat Rejection:

Turbine Work Output:

Vapor Quality at Turbine Exit:

Pump Work Input:

Cooling Capacity:

Total Heat Input:

Total Work Output:

First Law Efficiency:

Heat Source Flow Rate:

Heat Source Entrance Temperature:

Heat Source Exit Temperature:

Second law efficiency:
$523.9 \mathrm{~kJ} / \mathrm{s}$

$3.5 \mathrm{~kJ} / \mathrm{s}$

$482.5 \mathrm{~kJ} / \mathrm{s}$

$81.77 \mathrm{~kW}$

$90 \%$

$3.5 \mathrm{~kW}$

$36.8 \mathrm{~kW}$

$527.4 \mathrm{~kJ} / \mathrm{s}$

$81.77 \mathrm{~kW}$

$22.49 \%$

$2.211 \mathrm{~kg} / \mathrm{s}$

$440 \mathrm{~K}$

$384.4 \mathrm{~K}$

$43.13 \%$ 


\section{Resource Temperature of $360 \mathrm{~K}$}

This heat source temperature is within the range of flat-plate solar collectors and solar ponds. The cycle is optimized in terms of second law thermal efficiency. The optimum working condition for this heat source is listed in Table 4.4. At this heat source temperature, the optimum working condition does carry cooling capacity and the optimum concentration for ammonia strong solution is at a medium value, 0.67 .

Table 4.4 Optimum Working Condition

\begin{tabular}{|c|c|c|c|c|c|c|}
\hline Point & $\mathrm{T}(\mathrm{K})$ & $\mathrm{P}(\mathrm{bar})$ & $\mathrm{h}(\mathrm{kJ} / \mathrm{kg})$ & $\mathrm{s}(\mathrm{kJ} / \mathrm{kg} . \mathrm{K})$ & $\mathrm{X}$ & $\begin{array}{c}\text { Flow Rate } \\
(\mathrm{kg} / \mathrm{s})\end{array}$ \\
\hline 1 & 295.0 & 5.5 & -105.1 & 0.1989 & 0.6733 & 1.0000 \\
\hline 2 & 295.1 & 13.0 & -104.1 & 0.1989 & 0.6733 & 1.0000 \\
\hline 3 & 325.2 & 13.0 & 45.7 & 0.6805 & 0.6733 & 1.0000 \\
\hline 4 & 333.1 & 13.0 & 1371.9 & 4.4956 & 0.9935 & 0.2066 \\
\hline 5 & 311.1 & 13.0 & 102.9 & 0.5923 & 0.8949 & 0.0110 \\
\hline 6 & 311.1 & 13.0 & 1302.7 & 4.2793 & 0.9990 & 0.1956 \\
\hline 7 & 311.1 & 13.0 & 1302.7 & 4.2793 & 0.9990 & 0.1956 \\
\hline 8 & 280.6 & 5.5 & 1195.5 & 4.2793 & 0.9990 & 0.1956 \\
\hline 9 & 285.0 & 5.5 & 1278.3 & 4.5730 & 0.9990 & 0.1956 \\
\hline 10 & 333.1 & 13.0 & 45.2 & 0.7305 & 0.5942 & 0.8044 \\
\hline 11 & 300.1 & 13.0 & -106.8 & 0.2500 & 0.5942 & 0.8044 \\
\hline 12 & 300.2 & 5.5 & -106.8 & 0.2532 & 0.5942 & 0.8044 \\
\hline
\end{tabular}

Boiler Heat Input:

Superheat Input:

Absorber Heat Rejection:

Turbine Work Output:

Vapor Quality at Turbine Exit:

Pump Work Input:

Cooling Capacity:

Total Heat Input:

Total Work Output:

First Law Efficiency:
$272.9 \mathrm{~kJ} / \mathrm{s}$

$0 \mathrm{~kJ} / \mathrm{s}$

$269.1 \mathrm{~kJ} / \mathrm{s}$

$21.0 \mathrm{~kW}$

$93.93 \%$

$1.0 \mathrm{~kW}$

$16.2 \mathrm{~kW}$

$272.9 \mathrm{~kJ} / \mathrm{s}$

$19.99 \mathrm{~kW}$

$13.26 \%$ 
Heat Source Flow Rate:

Heat Source Entrance Temperature:

$2.183 \mathrm{~kg} / \mathrm{s}$

Heat Source Exit Temperature:

$360 \mathrm{~K}$

Second law efficiency:

$330.2 \mathrm{~K}$

$54.22 \%$ 


\section{CHAPTER 4 OPTIMIZATION OF AMMONIA-BASED COMBINED POWER/COOLING CYCLE}

In general, optimization is a way to find the best solution to a problem of any kind. We do a lot of optimization in our daily life. For example, we always search for the best product, which meets our demands, with the lowest possible price. In engineering, optimization is a very powerful tool. There is usually more than one acceptable solution to an engineering problem. The goal of an engineer is to find the best one. When there are more than a few acceptable solutions, it may be simply impossible to compare them by hand. More sophisticated mathematical methods have been developed to help find the best solution.

\section{Introduction to Optimization}

\section{Mathematical Formulation}

Mathematically speaking, optimization is the minimization or maximization of a function subject to a set of constraints on its variables. For a thermodynamic cycle, the optimization objective usually is to find the maximum efficiency or work output. Constraints would be like approach temperatures in a heat exchanger. Engineering optimization problems, despite their diversity, have amazingly the same mathematical formulation. It can be written as: 


$$
\begin{array}{lc}
\min & f(\boldsymbol{x}) \\
\text { s.t. } & \boldsymbol{h}(\boldsymbol{x})=0 \\
& \boldsymbol{g}(\boldsymbol{x}) \leq 0
\end{array}
$$

Where

$\boldsymbol{x}$ is the vector of a set of continuous real variables;

$f$ is the objective function, a function of $x$ that we want to minimize;

$\boldsymbol{h}(\boldsymbol{x})=0$ and $\boldsymbol{g}(\boldsymbol{x}) \leq 0$ are sets of equality constraints and inequality constraints, respectively.

If an optimization problem is to maximize rather than minimize $f$, we can easily accommodate this change by minimizing $-f$ in the formulation (4.1). A well-defined optimization problem features a number of variables greater than the number of equality constraints, which implies that there exist degrees of freedom upon which we optimize.

Formulation (4.1) contains a number of classes of optimization problems, by appropriate consideration or elimination of its elements. If a problem doesn't have constraints on the variables, then formulation (4.1) becomes an unconstrained optimization problem. Otherwise it's a constrained optimization problem. Among constrained optimization problems, if both the objective function and all the constraints are linear functions of $\boldsymbol{x}$, then formulation (4.1) becomes a linear programming (LP) problem. If at least one of the constraints or the objective function is nonlinear function, formulation (4.1) becomes a nonlinear programming (NLP) problem. A special case of constrained optimization is bound-constrained optimization. In this special case, all constraints are boundary constraints on $\boldsymbol{x}(\boldsymbol{L} \leq \boldsymbol{x} \leq \boldsymbol{U})$. 


\section{Optimality Conditions}

Before we start to search for the optimum, we need to answer a very basic question. How do we tell if a point is an optimum or not? Apparently, a condition or conditions are required to make such judgement. That condition or conditions are termed as optimality conditions. This section presents the necessary and sufficient optimality conditions for unconstrained and constrained optimization.

\section{Unconstrained optimization}

An unconstrained optimization problem deals with the search for a minimum of a function $f(x)$ of $\mathrm{n}$ real variables $\boldsymbol{x}=\left(x_{1}, x_{2}, \ldots, x_{\mathrm{n}}\right)$. For an unconstrained optimization problem, formulation (4.1) is simplified as:

$$
\min f(x)
$$

Each of the real variables $x_{i}$ is allowed to take any value from - $\infty$ to $\infty$.

Unconstrained optimization plays an important role in the optimization applications. It is not only because there are some applications which inherently are unconstrained, but also because constrained optimization algorithms are often the extensions of unconstrained optimization algorithms. The necessary and sufficient optimality conditions for an unconstrained optimization problem are:

\section{Theorem 4.1}

Let $f(x)$ be a twice differentiable function in $R^{\mathrm{n}}$ at $\boldsymbol{x}^{*}$. Then $\boldsymbol{x}^{*}$ is a local minimum if and only if 
i. $\nabla f\left(x^{*}\right)=0$, and

ii. The Hessian matrix $H\left(x^{*}\right)$, given by

$$
H\left(\boldsymbol{x}^{*}\right)=\frac{\partial^{2} f}{\partial x_{i} \partial x_{j}}\left(\boldsymbol{x}^{*}\right)
$$

is positive semidefinite; that is, $y^{T} H\left(x^{*}\right) y \geq 0$ for all $y \in R^{n}$.

When there's only one variable, the above necessary and sufficient conditions are simplified into a more familiar format:

i. $f^{\prime}(x)=0$;

ii. $f^{\prime \prime}(x) \geq 0$.

\section{Constrained optimization}

A constrained optimization problem deals with the search for a minimum of a function $f(x)$ of $\mathrm{n}$ real variables $\boldsymbol{x}=\left(x_{1}, x_{2}, \cdots, x_{\mathrm{n}}\right)$ subject to a set of equality constraints $\boldsymbol{h}(\boldsymbol{x})=0\left(h_{i}(\boldsymbol{x})=0, i=1,2, \cdots, \boldsymbol{m}\right)$, and a set of inequality constraints $\boldsymbol{g}(\boldsymbol{x}) \leq 0\left(g_{J}(\boldsymbol{x}) \leq 0\right.$, $j=1,2, \cdots, p)$. It has the same form of formulation (4.1), which is rewritten here:

$$
\begin{array}{ll}
\min & f(x) \\
\text { s.t. } & \boldsymbol{h}(\boldsymbol{x})=0 \\
& \boldsymbol{g}(\boldsymbol{x}) \leq 0
\end{array}
$$

If a point $\bar{x}$ satisfies the equality and inequality constraints in formulation (4.1), then it is a feasible point of the problem. Among inequality constraints, an inequality constraint $g_{j}(x)$ is called active at a feasible point $\bar{x}$ if $g_{j}(\bar{x})=0$ and inactive if $g_{j}(\bar{x})<0$. 
Compared with unconstrained optimization, constrained optimization is much more complicated. The key idea in developing necessary and sufficient optimality conditions for constrained optimization problems is to transform them into unconstrained problems and then apply the optimality conditions of unconstrained problems (Nocedal and Wright, 1999). The transformation involves the introduction of an auxiliary function, called the Lagrange function $L(\boldsymbol{x})$, defined as

$$
L(\boldsymbol{x}, \lambda, \mu)=f(\boldsymbol{x})+\lambda^{T} \boldsymbol{h}(\boldsymbol{x})+\boldsymbol{\mu}^{T} \boldsymbol{g}(\boldsymbol{x}), \quad \boldsymbol{\mu} \geq 0
$$

Where $\lambda^{T}=\left(\lambda_{1}, \lambda_{2}, \cdots, \lambda_{m}\right)$ and $\mu^{T}=\left(\mu_{1}, \mu_{2}, \cdots, \mu_{p}\right)$ are the Lagrange multipliers associated with the equality and inequality constraints, respectively. The multipliers $\lambda$ can be any real numbers, while the multipliers $\mu$ must be nonnegative.

Lagrange multipliers in formulation (4.4) are not just a group of coefficients. They have an important special meaning in constrained optimization. Each Lagrange multiplier for a given constraint indicates how much the Lagrange function $L$ will change for a differential change of that constraint. Considering a simple problem with only one equality constraint:

$$
\begin{array}{ll}
\min & f(x) \\
\text { s.t. } & h(x)=e
\end{array}
$$

Then its Lagrange function $L(\boldsymbol{x}, \lambda)$ is:

$$
L(x, \lambda)=f(x)+\lambda[h(x)-e]
$$

Take a derivative of the Lagrange function $L(x, \lambda)$ with respect to $e$ :

$$
\frac{\partial L}{\partial e}=-\lambda
$$


Therefore, the change of $L$ with the constant e is proportional to the Lagrange multiplier. Since the Lagrange function $L$ is equal to the objective function $f$ at the optimum, we can say that the Lagrange multipliers indicate the sensitivity of the objective function $f(x)$ with respect to perturbations on the constraints.

With the introduction of Lagrange function, a constrained optimization problem is transformed into an unconstrained problem according to duality theory (Floudas, 1995):

$$
\min _{x, \lambda, \mu \geq 0} L(\boldsymbol{x}, \lambda, \mu)=f(\boldsymbol{x})+\lambda^{T} \boldsymbol{h}(\boldsymbol{x})+\boldsymbol{\mu}^{T} \boldsymbol{g}(\boldsymbol{x})
$$

The necessary optimality conditions are given as below:

\section{Theorem 4.2 Karush-Kuhn-Tucker Necessary Conditions}

Let $f(\boldsymbol{x})$ and $\boldsymbol{g}(\boldsymbol{x})$ be differentiable at $\boldsymbol{x}^{*}$ and $\boldsymbol{h}(\boldsymbol{x})$ have continuous first partial derivatives at $x^{*}$. If $x^{*}$ is a local optimum of problem (4.1) and one constraint qualification ${ }^{1}$ is satisfied. Then there exist Lagrange multipliers $\lambda, \mu$ :

$$
\begin{aligned}
\nabla f\left(\boldsymbol{x}^{*}\right)+\lambda^{T} \nabla \boldsymbol{h}\left(\boldsymbol{x}^{*}\right)+\boldsymbol{\mu}^{T} \nabla \boldsymbol{g}\left(\boldsymbol{x}^{*}\right) & =0 \\
\boldsymbol{h}\left(\boldsymbol{x}^{*}\right) & =0 \\
\boldsymbol{g}\left(\boldsymbol{x}^{*}\right) & \leq 0 \\
\mu_{j} g_{j}\left(\boldsymbol{x}^{*}\right) & =0 \quad j=1,2, \cdots, p \\
\mu_{j} & \geq 0 \quad j=1,2, \cdots, p .
\end{aligned}
$$

A point which satisfies Karush-Kuhn-Tucker conditions is called a KKT point.

\footnotetext{
${ }^{1}$ First-order and second-order constraint qualifications are satisfied if the gradients of all equality and active inequality constraints are linearly independent.
} 
However, theorem 4.2 only utilizes the first-order information of the objective function and constraints. The second order information, the curvature of the functions, is not considered. Fiacco and McCormick (1968) demonstrated that first-order information is not complete with their famous example:

$$
\begin{aligned}
& \min \quad\left(x_{1}-1\right)^{2}+x_{2}^{2} \\
& \text { s.t. } \quad x_{1}-\frac{x_{2}^{2}}{k} \leq 0
\end{aligned}
$$

Where the values of the parameter $k>0$, for which $(0,0)$ is a local minimum, are sought.

In this example, we only have one constraint. Its gradient

$$
\left(1,-\frac{2 x_{2}}{k}\right)
$$

is always nonzero. So it's linearly independent by itself. A constrain qualification is satisfied. At $(0,0)$, the gradient KKT conditions are:

$$
\begin{aligned}
& \left(\begin{array}{c}
-2 \\
0
\end{array}\right)+\mu\left(\begin{array}{l}
1 \\
0
\end{array}\right)=\left(\begin{array}{l}
0 \\
0
\end{array}\right) \\
& \therefore \quad \mu=2 \geq 0
\end{aligned}
$$

The constraints KKT conditions are satisfied since:

$$
x_{1}-\frac{x_{2}^{2}}{k}=0
$$

Therefore, KKT necessary conditions are satisfied. However, for $k=1$ the point $(0,0)$ is not a local minimum while for $k=4$ it is.

From the above example, we learn that in order to find a local optimum, second order information has to be taken into account. The second order necessary and sufficient conditions are presented: 


\section{Theorem 4.3 Second-Order Necessary Optimality Conditions}

Let $f(x), \boldsymbol{h}(\boldsymbol{x})$ and $\boldsymbol{g}(\boldsymbol{x})$ be twice continuously differentiable at $\boldsymbol{x}^{*}$. If $\boldsymbol{x}^{*}$ is a local optimum of problem (4.1) and the second order constraint qualification is satisfied ${ }^{1}$, and if there exist Lagrange multipliers $\lambda^{*}, \mu^{*}$ satisfying the KKT first-order necessary conditions:

$$
\begin{aligned}
\nabla f\left(\boldsymbol{x}^{*}\right)+\lambda^{* T} \nabla \boldsymbol{h}\left(\boldsymbol{x}^{*}\right)+\mu^{* T} \nabla \boldsymbol{g}\left(\boldsymbol{x}^{*}\right) & =0 \\
\boldsymbol{h}\left(\boldsymbol{x}^{*}\right) & =0 \\
\boldsymbol{g}\left(\boldsymbol{x}^{*}\right) & \leq 0 \\
\mu_{j}^{*} g_{j}\left(\boldsymbol{x}^{*}\right) & =0 \quad j=1,2, \cdots, p \\
\mu_{j}^{*} & \geq 0 \quad j=1,2, \cdots, p
\end{aligned}
$$

and if for every nonzero vector $z$ :

$$
\begin{array}{ll}
z^{T} \nabla h_{i}\left(x^{*}\right)=0 & i=1,2, \cdots, m, \text { and } \\
z^{T} \nabla g_{i}\left(x^{*}\right)=0 & j \in J \equiv\left\{j: g_{j}\left(x^{*}\right)=0\right\}
\end{array}
$$

then,

$$
z^{T} \nabla^{2} L\left(x^{*}, \lambda^{*}, \mu^{*}\right) z \geq 0
$$

\section{Theorem 4.4 Second-Order Sufficient Optimality Conditions}

Let $f(x), \boldsymbol{h}(\boldsymbol{x})$ and $\boldsymbol{g}(\boldsymbol{x})$ be twice differentiable at $\boldsymbol{x}^{*}$. If there exist Lagrange multipliers $\lambda^{*}, \mu^{*}$ satisfying the KKT first-order necessary conditions:

$$
\begin{aligned}
\nabla f\left(\boldsymbol{x}^{*}\right)+\lambda *^{T} \nabla \boldsymbol{h}\left(\boldsymbol{x}^{*}\right)+\boldsymbol{\mu}^{*^{T}} \nabla \boldsymbol{g}\left(\boldsymbol{x}^{*}\right) & =0 \\
\boldsymbol{h}\left(\boldsymbol{x}^{*}\right) & =0
\end{aligned}
$$




$$
\begin{aligned}
\boldsymbol{g}\left(\boldsymbol{x}^{*}\right) & \leq 0 \\
\mu_{j}^{*} g_{j}\left(\boldsymbol{x}^{*}\right) & =0 \quad j=1,2, \cdots, p \\
\mu_{j}^{*} & \geq 0 \quad j=1,2, \cdots, p
\end{aligned}
$$

If for every nonzero vector $z$ :

$$
\begin{array}{ll}
z^{T} \nabla h_{i}\left(x^{*}\right)=0 & i=1,2, \cdots, m, \text { and } \\
z^{T} \nabla g_{i}\left(x^{*}\right)=0 & j \in J_{1} \equiv\left\{j: g_{j}\left(x^{*}\right)=0, \mu_{j}>0\right\} \\
z^{T} \nabla g_{i}\left(x^{*}\right) \leq 0 & j \in J_{2} \equiv\left\{j: g_{j}\left(x^{*}\right)=0 \quad \mu_{j}=0\right\}
\end{array}
$$

it follows that

$$
z^{T} \nabla^{2} L\left(x^{*}, \lambda^{*}, \mu^{*}\right) z>0
$$

then, $\boldsymbol{x}^{*}$ is a strict local minimum of problem (4.1).

\section{Generalized Reduced Gradient Algorithm}

The optimality conditions presented in the previous section provide the theoretical basis for the development of different optimization algorithms. Generalized Reduced Gradient (GRG) Algorithm is one of the most commonly used algorithms for solving constrained optimization.

As stated in the previous section, a constrained optimization problem can be expressed as:

$$
\begin{array}{lc}
\min & f(\boldsymbol{x}) \\
\text { s.t. } & \boldsymbol{h}(\boldsymbol{x})=0 \\
& \boldsymbol{g}(\boldsymbol{x}) \leq 0
\end{array}
$$


Among inequality constraints, they are subdivided into two categories: boundary constraints ( $L_{i} \leq x_{i} \leq U_{i}$, one of $L_{i}, U_{i}$ could be infinite) and function inequality constraints. Boundary constraints are separated from other inequality constraints because they are treated differently in determining the step length in a search direction as we'll see later. Function inequality constraints can be transformed into equality constraints by adding slack variables. For example, an inequality constraint

$$
g_{j}(x) \leq 0
$$

By adding slack variable $x_{n+j}$, it can be redefined as

$$
h_{m+j}(x)=g_{j}(x)+x_{n+j}=0
$$

Where $x_{n+j} \geq 0$. Contrary to slack variables, variable $\mathrm{x}_{1}, \mathrm{x}_{2}, \ldots, \mathrm{x}_{\mathrm{n}}$ are called natural variables. Therefore, an inequality constraint, which is the function of natural variables, is transformed into an equality constraint, which is the function of natural variables plus a slack variable, and a boundary constraint on that slack variable.

By treating boundary constraints separately and transforming inequality constraints to equality constraints, problem (4.1) is converted to the following equivalent form:

$$
\begin{array}{lll}
\min & f(\boldsymbol{x}) & \boldsymbol{x}=\left[x_{1}, x_{2}, \cdots, x_{\mathrm{n}}\right]^{\mathrm{T}} \\
\text { s.t. } & h_{j}(\boldsymbol{x})=0 & j=1, \cdots, m \\
& L_{i} \leq x_{i} \leq U_{i} & i=1, \cdots, n
\end{array}
$$


However, keep in mind that $\mathrm{m}, \mathrm{n}$ are different numbers here with those in formulation (4.1) and $\boldsymbol{x}$ is the set of natural variables and slack variables. (4.6) is the formulation we are going to use for describing GRG algorithm.

The generalized reduced gradient algorithm is based on a very basic optimization procedure which transforms a constrained optimization problem into one that is unconstrained or bound-constrained. It classes the variables as independent and dependent, and substitutes into the objective function the expressions obtained from the equality constraints in independent variables for the dependent variables. This essentially reduces the original problem to an unconstrained or bound-constrained problem of reduced dimension (Lasdon, et al., 1978).

In terms of dependent and independent variables, the constraints in formulation (3.6) can be written as

$$
h(x)=h\left(x_{1}, x_{\mathbf{D}}\right)=0
$$

Where $\boldsymbol{x}_{\mathrm{I}}$ are the set of independent variables, $\boldsymbol{x}_{\mathrm{D}}$ are the set of dependent variables. $\boldsymbol{x}_{\mathrm{D}}$ are solved such that

$$
x_{\mathrm{D}}=\varphi\left(\boldsymbol{x}_{\mathbf{I}}\right)
$$

According to the formulation (4.6), the number of $x_{\mathbf{I}}$ is (n-m) and $\boldsymbol{x}_{\mathbf{D}}$ is $\mathrm{m}$. Thus the objective function can be written as

$$
f(x)=f\left(x_{\mathbf{I}}, x_{\mathbf{D}}\right)=f\left(x_{\mathbf{I}}, \varphi\left(x_{\mathbf{I}}\right)\right)=\mathrm{F}\left(x_{\mathbf{I}}\right)
$$

The original problem is now transformed into a simpler reduced problem of $(n-m)$ dimension:

$\min F(x)$

$$
\text { s.t. } \quad L \leq x \leq U
$$


Where $\boldsymbol{L}$ and $\boldsymbol{U}$ are the bound vectors for $\boldsymbol{x}$. The function $\mathrm{F}(\boldsymbol{x})$ is called the reduced objective.

The concept of the reduced gradient is best described by the following simple example (Edgar and Himmelblau, 1988):

$$
\begin{array}{ll}
\min & f\left(x_{1}, x_{2}\right) \\
\text { s.t. } & h\left(x_{1}, x_{2}\right)=0
\end{array}
$$

The total derivatives of each function are:

$$
\begin{aligned}
& d f(\boldsymbol{x})=\frac{\partial f(\boldsymbol{x})}{\partial x_{1}} d x_{1}+\frac{\partial f(\boldsymbol{x})}{\partial x_{2}} d x_{2} \\
& d h(\boldsymbol{x})=\frac{\partial h(\boldsymbol{x})}{\partial x_{1}} d x_{1}+\frac{\partial h(\boldsymbol{x})}{\partial x_{2}} d x_{2}=0
\end{aligned}
$$

If $x_{1}$ is designated to be the dependent variable, $x_{2}$ to be the independent variable, then $\mathrm{d} x_{1}$ can be eliminated from Eq. (4.9) by use of Eq. (4.10) since

$$
d x_{1}=-\frac{\left[\partial h(x) / \partial x_{2}\right]}{\left[\partial h(x) / \partial x_{1}\right]} d x_{2}
$$

Therefore,

$$
d f(\boldsymbol{x})=\left\{\left[\frac{\partial f(\boldsymbol{x})}{\partial x_{2}}\right]-\left[\frac{\partial f(\boldsymbol{x})}{\partial x_{1}}\right]\left[\frac{\partial h(\boldsymbol{x})}{\partial x_{1}}\right]^{-1}\left[\frac{\partial h(\boldsymbol{x})}{\partial x_{2}}\right]\right\} d x_{2}
$$


The expression in the brace is called the reduced gradient. Of course, in this problem the reduced gradient contains only one element because there is only one independent variable. The general form of the reduced gradient is:

$$
g_{R}^{T}=\left[\frac{\partial f(\boldsymbol{x})}{\partial \boldsymbol{x}_{\mathbf{I}}}\right]^{T}-\left[\frac{\partial f(\boldsymbol{x})}{\partial \boldsymbol{x}_{\mathrm{D}}}\right]^{T}\left[\frac{\partial \boldsymbol{h}(\boldsymbol{x})}{\partial \boldsymbol{x}_{\mathrm{D}}}\right]^{-1}\left[\frac{\partial \boldsymbol{h}(\boldsymbol{x})}{\partial \boldsymbol{x}_{\mathbf{I}}}\right]
$$

The generalized reduced-gradient algorithm can be stated in five basic steps (Edgar and Himmelblau, 1988). In the text, $\boldsymbol{x}_{\mathbf{I}}$ and $\boldsymbol{x}_{\mathrm{D}}$ bear superscripts $\mathrm{k}$ since their components may well change from stage to stage. Also to simplify the notation, we denote $f\left(x^{\mathrm{k}}\right)$ as $f^{\mathrm{k}}$ and $\boldsymbol{h}\left(\boldsymbol{x}^{\mathrm{k}}\right)$ as $\boldsymbol{h}^{\mathrm{k}}$.

\section{Step 1 Determine the search components for the independent variables.}

At stage $\mathrm{k}$, linearize the constraints at the feasible point $x^{\mathrm{k}}$ and compute the reduced gradient:

$$
\left(g_{R}{ }^{k}\right)^{T}=\left[\frac{\partial f^{k}}{\partial x_{\mathrm{I}}^{k}}\right]^{T}-\left[\frac{\partial f^{k}}{\partial \boldsymbol{x}_{\mathrm{D}}^{k}}\right]^{T}\left[\frac{\partial \boldsymbol{h}^{k}}{\partial \boldsymbol{x}_{\mathrm{D}}^{k}}\right]^{-1}\left[\frac{\partial \boldsymbol{h}^{k}}{\partial \boldsymbol{x}_{\mathrm{I}}^{k}}\right]
$$

Then the search direction components of the independent variables are established as:

$$
\Delta_{i}^{k}=-g_{R i}^{k}
$$

However, $\Delta_{i}^{k}$ is selected as 0 if $\mathrm{x}_{\mathrm{i}}$ is at one of its bounds and the step would exceed the bound. That is,

$$
\begin{aligned}
& \Delta_{i}^{k}=0 \quad \text { if } x_{i}^{k}=U_{\mathrm{i}} \quad g_{\mathrm{Ri}}<0 \\
& =L_{\mathrm{i}} \quad g_{\mathrm{Ri}}>0
\end{aligned}
$$




\section{Step 2 Determine the search components for the dependent variables.}

A desired search direction would be along the surface of the constraints. This is accomplished by finding the tangent to $\boldsymbol{h}(\boldsymbol{x})=0$ at the point $\left(\boldsymbol{x}_{\mathrm{I}}^{k}, \boldsymbol{x}_{\mathrm{D}}^{k}\right)$. That is,

$$
\begin{aligned}
& d \boldsymbol{h}=\frac{\partial \boldsymbol{h}}{\partial \boldsymbol{x}_{\mathrm{I}}} d \boldsymbol{x}_{\mathrm{I}}+\frac{\partial \boldsymbol{h}}{\partial \boldsymbol{x}_{\mathrm{D}}} d \boldsymbol{x}_{\mathrm{D}}=0 \\
& d \boldsymbol{x}_{\mathrm{D}}=-\left[\frac{\partial \boldsymbol{h}}{\partial \boldsymbol{x}_{\mathrm{D}}}\right]^{-1}\left[\frac{\partial \boldsymbol{h}}{\partial \boldsymbol{x}_{\mathrm{I}}}\right] d \boldsymbol{x}_{\mathrm{I}}
\end{aligned}
$$

Therefore, the search direction components of the dependent variables are:

$$
\Delta_{\mathrm{D}}^{k}=-\left[\frac{\partial \boldsymbol{h}^{k}}{\partial \boldsymbol{x}_{\mathrm{D}}^{k}}\right]^{-1}\left[\frac{\partial \boldsymbol{h}^{k}}{\partial \boldsymbol{x}_{\mathrm{I}}^{k}}\right] \Delta_{\mathrm{I}}^{k}
$$

\section{Step 3 Improving the value of the objective function.}

Minimize $f\left(\mathbf{x}_{\mathrm{I}}^{k}+\lambda \Delta_{\mathrm{I}}^{k}, \mathbf{x}_{\mathrm{D}}^{k}+\lambda \Delta_{\mathrm{D}}^{k}\right)$ with respect to $\lambda(\lambda>0)$ by a one-dimensional search. The new point is:

$$
\begin{aligned}
& \boldsymbol{x}_{\mathbf{I}}^{k+1}=\boldsymbol{x}_{\mathbf{I}}^{k}+\lambda^{k} \Delta_{\mathrm{I}}^{k} \\
& \widetilde{\boldsymbol{x}}_{\mathbf{D}}^{k+1}=\boldsymbol{x}_{\mathbf{D}}^{k}+\lambda^{k} \Delta_{\mathrm{D}}^{k} \quad \widetilde{\mathbf{x}}_{\mathbf{D}} \text { is a tentative point }
\end{aligned}
$$




\section{Step 4 Use Newton's method to regain feasibility of dependent variables.}

In general, some of the components of $\widetilde{\mathbf{x}}_{\mathbf{D}}^{k+1}$ are not feasible, hence $\boldsymbol{h}\left(\boldsymbol{x}_{I}^{k+1}, \widetilde{\boldsymbol{x}}_{D}^{k+1}\right) \neq 0$. In order to return from a point away from constraints back to a point satisfying the constraints, $\widetilde{\boldsymbol{x}}_{\mathbf{D}}^{k+1}$ is modified by Newton's method:

$$
\boldsymbol{x}_{\mathbf{D}}^{k+1}=\tilde{\boldsymbol{x}}_{\mathbf{D}}^{k+1}-\left[\frac{\partial \boldsymbol{h}\left(\boldsymbol{x}_{\mathbf{I}}^{k+1}, \widetilde{\boldsymbol{x}}_{\mathrm{D}}^{k+1}\right)}{\partial \boldsymbol{x}_{\mathrm{D}}^{k}}\right]^{-1} \boldsymbol{h}\left(\boldsymbol{x}_{\mathbf{I}}^{k+1}, \tilde{\boldsymbol{x}}_{\mathbf{D}}^{k+1}\right)
$$

Several iterations of Newton's method may be needed to find a feasible point. Figure 4.1 illustrates the move from $x^{k}$ to $\left(x_{\mathrm{I}}^{k+1}, \tilde{x}_{\mathrm{D}}^{k+1}\right)$ and then to $\left(x_{\mathrm{I}}^{k+1}, x_{\mathrm{D}}^{k+1}\right)$ by Newton's method.

Note: Newton's method finds the desired root $\boldsymbol{x}_{\mathbf{D}}^{k+1}$ with $\widetilde{\boldsymbol{x}}_{\mathbf{D}}^{\mathbf{k}+1}$ as the starting point. By expanding $\boldsymbol{h}\left(\boldsymbol{x}_{\mathbf{I}}^{k+1}, \boldsymbol{x}_{\mathbf{D}}^{k+1}\right)$ and equating it to zero, we get

$$
\boldsymbol{h}\left(\boldsymbol{x}_{\mathrm{I}}^{k+1}, \boldsymbol{x}_{\mathrm{D}}^{k+1}\right) \approx \boldsymbol{h}\left(\boldsymbol{x}_{\mathbf{I}}^{k+1}, \widetilde{\boldsymbol{x}}_{\mathrm{D}}^{k+1}\right)+\frac{\partial \boldsymbol{h}\left(\boldsymbol{x}_{\mathbf{I}}^{k+1}, \widetilde{\boldsymbol{x}}_{\mathrm{D}}^{k+1}\right)}{\partial \boldsymbol{x}_{\mathrm{D}}^{k}}\left(\boldsymbol{x}_{\mathrm{D}}^{k+1}-\widetilde{\boldsymbol{x}}_{\mathbf{D}}^{k+1}\right)=0
$$

\section{Step 5 Procedure on lack of convergence of Newton's method.}

(a) If $\boldsymbol{x}^{k+1}$ is a feasible point and $f\left(x^{k+1}\right)<f\left(x^{k}\right)$, adopt $\boldsymbol{x}^{k+1}$, and start with step 1 again.

(b) If $\boldsymbol{x}^{k+1}$ is a feasible point and $f\left(x^{k+1}\right)>f\left(x^{k}\right)$, reduce $\lambda$ by a factor and start step 4 over again.

(c) If Newton's method fails to find a feasible point after certain times, reduce $\lambda$ by a factor and start step 4 over again. 


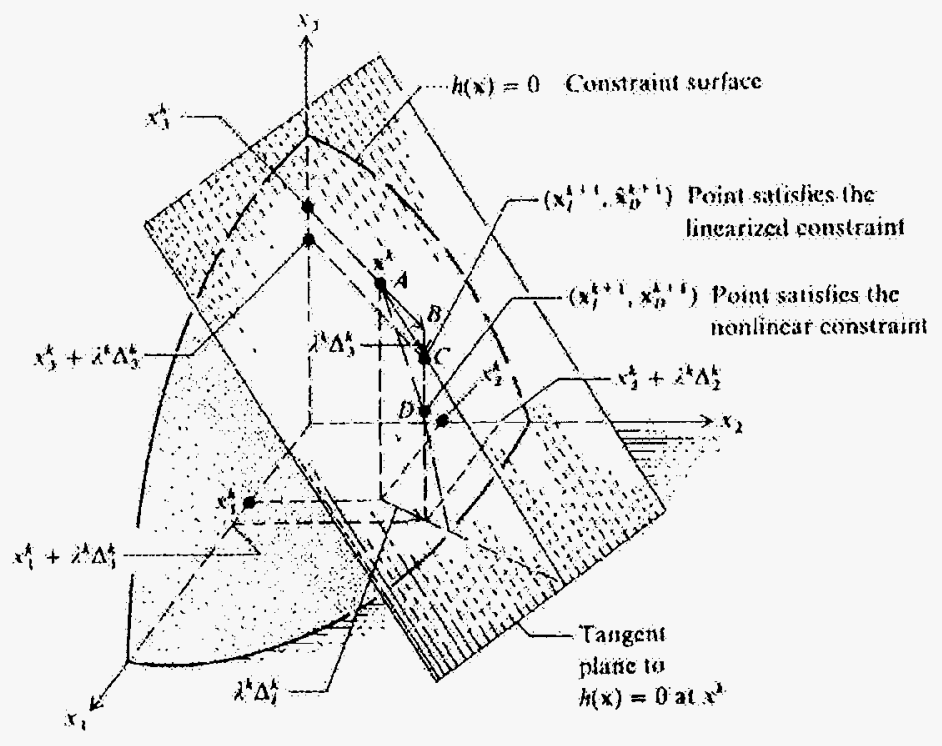

Figure 4.1 Illustration Of Using Newton's Method To Regain Feasibility

(d) If neither (a), (b) nor (c) occur, or (b) and (c) are unsuccessful after several passes, change the basis by exchanging a dependent variable with a former independent variable. This process is termed a change of basis.

Theoretically, the stopping condition for optimization is when KKT conditions are satisfied. However, in practice, we can use a simpler condition:

$$
\left|\frac{f\left(x^{k+1}\right)-f\left(x^{k}\right)}{f\left(x^{k}\right)}\right|<\varepsilon
$$

Where $\varepsilon$ is a small number.

A numeric example is given as following to illustrate the GRG algorithm. 


\section{EXAMPLE:}

$$
\begin{array}{ll}
\max & f(x)=\left(2 x_{1}-0.5 x_{1}^{2}\right)+\left(3 x_{2}-0.5 x_{2}^{2}\right) \\
\text { s.t. } & h(\mathbf{x})=x_{1}^{2}+x_{2}^{2}+x_{3}-1=0 \\
& -2 \leq x_{j} \leq 2 \quad j=1,2,3
\end{array}
$$

Solution: We minimize $-f(x)$. In the text below, $f(\boldsymbol{x})$ actually refers to $-f(\boldsymbol{x})$.

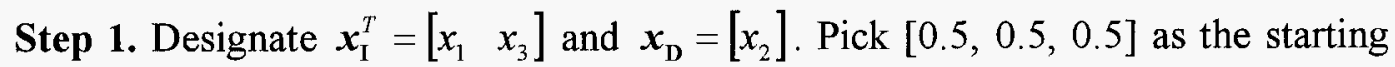
point. The reduced gradient is:

$$
g_{R}^{T}=\left[\frac{\partial f(\boldsymbol{x})}{\partial \boldsymbol{x}_{\mathbf{1}}}\right]^{T}-\left[\frac{\partial f(\boldsymbol{x})}{\partial \boldsymbol{x}_{\mathbf{D}}}\right]^{T}\left[\frac{\partial \boldsymbol{h}(\boldsymbol{x})}{\partial \boldsymbol{x}_{\mathbf{D}}}\right]^{-1}\left[\frac{\partial \boldsymbol{h}(\boldsymbol{x})}{\partial \boldsymbol{x}_{\mathbf{I}}}\right]
$$

where

$$
\begin{array}{lc}
\frac{\partial f(\boldsymbol{x})}{\partial \boldsymbol{x}_{\mathrm{I}}}=-\left[\begin{array}{c}
2-x_{1} \\
0
\end{array}\right] & \frac{\partial f(\boldsymbol{x})}{\partial \boldsymbol{x}_{\mathbf{D}}}=-\left(3-x_{2}\right) \\
\frac{\partial \boldsymbol{h}(\boldsymbol{x})}{\partial \boldsymbol{x}_{\mathrm{I}}}=\left[\begin{array}{ll}
2 x_{1} & 1
\end{array}\right] & \frac{\partial \boldsymbol{h}(\boldsymbol{x})}{\partial \boldsymbol{x}_{\mathbf{D}}}=2 x_{2} \\
\left(g_{R}^{0}\right)^{T}=-\left[\begin{array}{ll}
2-x_{1}^{0} & 0
\end{array}\right]-\left[-\left(3-x_{2}^{0}\right)\right]\left[\frac{1}{2 x_{2}^{0}}\right]\left[\begin{array}{ll}
2 x_{1}^{0} & 1
\end{array}\right]=\left[\begin{array}{ll}
1 & 2.5
\end{array}\right]
\end{array}
$$

As the result, the search direction of the independent variable is,

$$
\Delta_{\mathrm{I}}^{0}=-g_{R}^{0}=\left[\begin{array}{c}
-1 \\
-2.5
\end{array}\right]
$$

Step 2. The search direction of the dependent variable is:

$$
\Delta_{\mathrm{D}}^{0}=-\left[\frac{\partial \boldsymbol{h}^{0}}{\partial \boldsymbol{x}_{\mathrm{D}}^{0}}\right]^{-1}\left[\frac{\partial \boldsymbol{h}^{0}}{\partial \boldsymbol{x}_{\mathbf{I}}^{0}}\right] \Delta_{\mathbf{I}}^{0}=-\left[\frac{1}{2 x_{2}^{0}}\right]\left[\begin{array}{ll}
2 x_{1}^{0} & 1
\end{array}\right] \Delta_{\mathbf{I}}^{0}=3.5
$$

Step 3. From above, we get 


$$
\begin{aligned}
& \Delta^{0}=\left[\begin{array}{c}
-1 \\
3.5 \\
-2.5
\end{array}\right] \\
& f\left(x^{0}+\lambda \Delta^{0}\right)=-\left\{\left[2(0.5-\lambda)-0.5(0.5-\lambda)^{2}\right]+\left[3(0.5+3.5 \lambda)-0.5(0.5+3.5 \lambda)^{2}\right]\right\}
\end{aligned}
$$

Minimize $f\left(x^{0}+\lambda \Delta^{0}\right)$ with respect to $\lambda$ by setting $(\partial f / \partial \lambda)=0$ and solving the resulting equation. We get

$$
\begin{aligned}
& \lambda^{0}=0.547 \\
& \boldsymbol{x}_{\mathrm{I}}^{1}=\left[\begin{array}{l}
0.5 \\
0.5
\end{array}\right]+0.547\left[\begin{array}{c}
-1 \\
-2.5
\end{array}\right]=\left[\begin{array}{l}
-0.047 \\
-0.867
\end{array}\right] \\
& \tilde{\boldsymbol{x}}_{\mathrm{D}}^{1}=[0.5]+0.547[3.5]=2.415
\end{aligned}
$$

Step 4. Check if $h(x)=0$,

$$
h^{1}=(-0.047)^{2}+(2.415)^{2}+(-0.867)-1=3.967 \neq 0
$$

Constraint is not satisfied. Applying Newton's method to modify $\tilde{x}_{2}^{1}$ :

$$
\begin{aligned}
& \frac{\partial h^{1}}{\partial x_{2}^{1}}=2 \tilde{x}_{2}^{1}=4.829 \\
& \hat{x}_{2}^{1}=2.415-\left(\frac{1}{4.829}\right)(3.967)=1.594
\end{aligned}
$$

Successively applying Newton's method, starting each time with the previously found $\hat{x}_{2}^{1}$, until a feasible $x_{2}^{1}$ is found. We give the result here:

$$
x_{2}^{1}=1.366
$$

\section{Step 5.}

$$
\begin{aligned}
& f\left(x^{0}\right)=-2.25 \\
& f\left(x^{1}\right)=-3.07
\end{aligned}
$$


$\therefore \quad f\left(\boldsymbol{x}^{1}\right)<f\left(\boldsymbol{x}^{0}\right)$

$x^{1}$ is a better point and adopted as new starting point. New search starts with step 1 again. Normally, the starting point, $x^{0}$, is a feasible solution. But that's not necessary. If the starting point, $x^{0}$, is not feasible, the optimization is done in two phases. In phases I, $x^{0}$ is made feasible by adding artificial variables to satisfy the equality constraints, then forcing them to zero values thus finding a new feasible point (Hwang, et al., 1972). Let an artificial variable, $x_{\mathrm{n}+\mathrm{i}}$, satisfy the condition,

$$
0 \leq x_{n+i} \leq \infty
$$

If, at the starting point $\boldsymbol{x}^{0}$, the $i$ th constraint violates the equality condition by being greater than zero, i.e., $h_{\mathrm{i}}\left(\boldsymbol{x}^{0}\right)>0$, it is made feasible by subtracting the artificial variable, $x_{n+\mathrm{i}}$,

$$
h_{\mathrm{i}}\left(x^{0}\right)-x_{\mathrm{n}+\mathrm{i}}=0
$$

Likely, if $h_{\mathrm{i}}\left(x^{0}\right)<0$, it is made feasible by adding the artificial variable,

$$
h_{\mathrm{i}}\left(x^{0}\right)+x_{\mathrm{n}+\mathrm{i}}=0
$$

The objective function to be minimized in phase I becomes the sum of all artificial variables,

$$
\min \sum_{i \in N E} x_{n+i} \quad N E=\left\{i \mid h_{i}(\boldsymbol{x}) \neq 0\right\}
$$

The artificial variables are forced to zero by minimization. When all of the artificial variables are zero, the solution, $\boldsymbol{x}$, becomes feasible. The second phase optimization begins with this new feasible solution. 


\section{Description of the Problem}

\section{Variable Temperature Heat Source}

For a given heat source fluid and a fixed ambient temperature $\mathrm{T}_{0}$, the maximum useful work (per unit weight of heat source fluid) is a function of only $T_{h s}^{i n}$, the heat source temperature:

$$
w_{\max }=\left(h_{h s}^{\text {in }}-h_{0}\right)-T_{0}\left(s_{h s}^{\text {in }}-s_{0}\right)
$$

Where $h_{h s}^{\text {in }}$ is the inlet enthalpy of the heat source fluid;

$s_{h s}^{i n}$ is the inlet entropy of the heat source fluid;

$h_{0}$ is the enthalpy of the heat source fluid at ambient temperature;

$s_{0}$ is the entropy of the heat source fluid at ambient temperature.

A plot of this value is given in Figure 4.2. The heat source fluid in Figure 4.2 is saturated water when its temperature is above $100^{\circ} \mathrm{C}$ and subcooled water at 1 atm when the temperature is below $100^{\circ} \mathrm{C}$.

Any real process will have nonreversible steps that will result in a net work output less than maximum useful work. These irreversibilities can be evaluated for each step of the conversion process. For example, any heat exchange step destroys availability due to the finite temperature difference between the fluids. Although big temperature difference favors the heat transfer process, thus requiring less heat transfer surface area, it will cause a degradation of availability and a corresponding loss in thermodynamic performance of the process. Optimal operation with the minimum irreversibility occurs when the heat capacities of both streams are constant. This situation produces a balanced exchanger in 


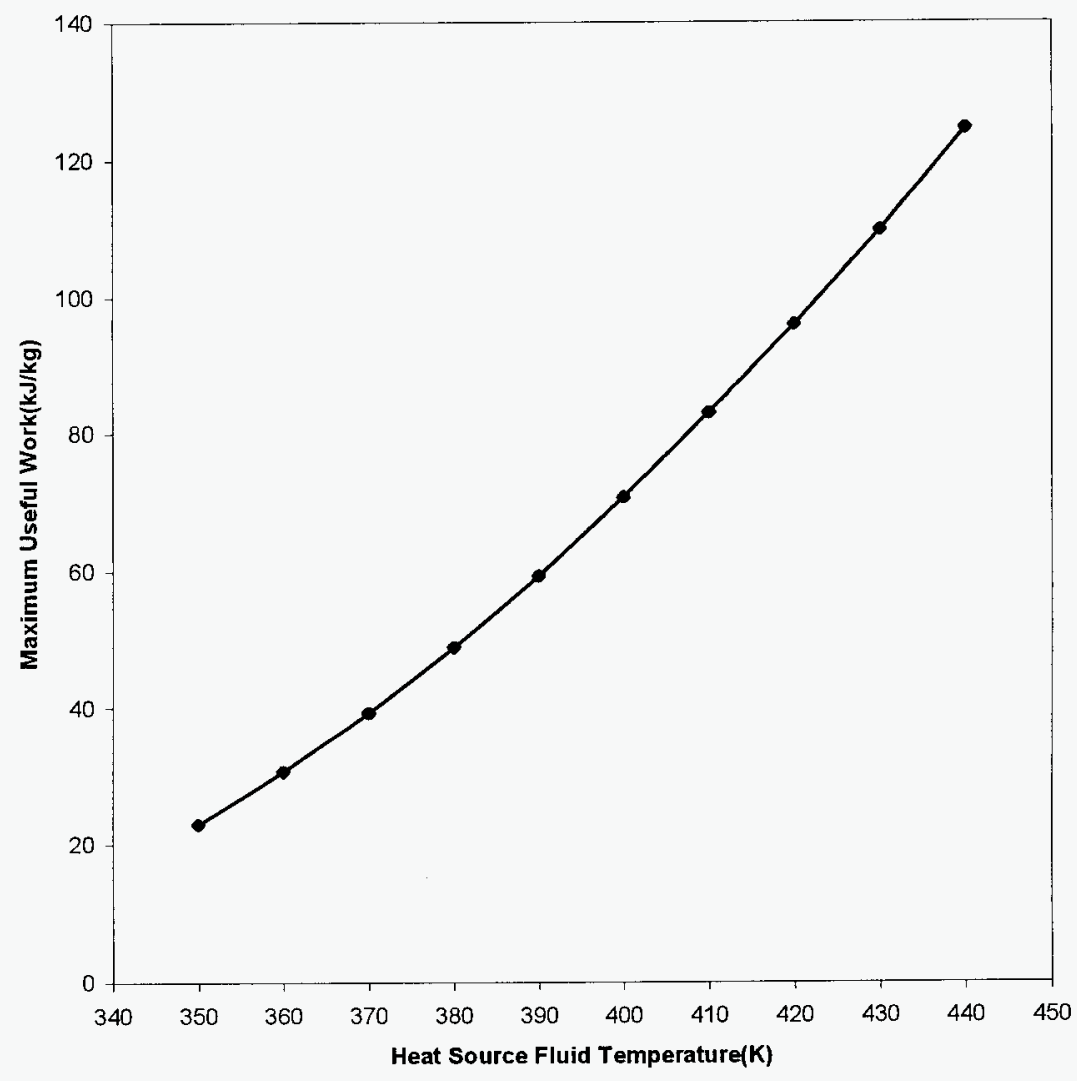

Figure 4.2 Maximum Useful Work Plotted as a Fuction of Heat Source Fluid Temperature

which the temperature difference $\Delta \mathrm{T}$ between streams can be kept uniform. In the heat exchanger, a "pinch" or minimum temperature difference occurs at the point where the liquid begins to evaporate. Efficient resource utilization will result when (Milora, 1976):

1. Most of the heat is extracted from the heat source fluid before disposal.

2. Temperature differentials across heat transfer surfaces are maintained at minimum practical levels.

3. Turbines and feed pumps are carefully designed for optimum efficiency.

4. Heat is rejected from the thermodynamic cycle at a temperature near the minimum ambient temperature. 
Quantitatively, one can define a cycle efficiency factor that is a measure of the fraction of the available energy of the heat source fluid that is converted into useful work. Second law thermal efficiency $\eta_{2}$, is defined in terms of net power output $\mathrm{W}_{\text {net, }}$, the heat source flow rate, $m_{h s,}$ and the availability:

$$
\eta_{2}=\frac{W_{n e t}}{m_{h s}\left[\left(h_{h s}^{i n}-h_{0}\right)-T_{0}\left(s_{h s}^{i n}-s_{0}\right)\right]}
$$

$\eta_{2}$ is a direct measure of the efficiency of resource utilization, because for a fixed $T_{h s}^{\text {in }}$, higher value of $\eta_{2}$ corresponds to lower flow rate of the source fluid for a given net power output. $\eta_{2}$ should not be confused with the first law thermal efficiency, $\eta_{1}$, which is a measure of how efficiently the transferred heat is converted into work.

$$
\eta_{1}=\frac{W_{n e t}}{m_{h s}\left(h_{h s}^{\text {in }}-h_{h s}^{\text {out }}\right)}
$$

Where $h_{h s}^{\text {out }}$ is the outlet enthalpy of the heat source fluid. The quality of the heat source and the availability of the disposed heat source fluid at temperatures above ambient are not considered in calculating $\eta_{1}$.

\section{Optimization Model for the Cycle}

Optimization is a very powerful tool in the engineering decision-making. A typical engineering problem can be expressed as follows: We have a process that can be represented by some equations and/or experimental data. We also have a performance criterion in mind, for example, to achieve maximum thermal efficiency. The goal of the 
optimization is to find an operating condition, which is represented by the values of the some variables, that yields the best performance.

Problem formulation is perhaps the most crucial step in resolving a problem that involves optimization. Problem formulation requires identifying a conceptual statement of a given application, and organizing them into a standard mathematical form. Namely

1. The objective function

\section{Constraints}

The objective function represents, in terms of the key variables, the process being analyzed. The constraints describe the interrelationships of the key variables. Constraints in optimization problems arise from physical bounds on the variables, empirical relations, physical laws, etc.

For this ammonia-based combined power/cooling cycle, our optimization objective is to find the maximum thermal efficiency. By looking into the cycle (Fig. 2.7), we can see that the high and low pressure of the cycle can be freely changed and have to be chosen by the designer. In addition, the temperatures of the absorber, boiler, rectifier and superheater, are also free variables.

From the previous parametric analysis, we have concluded higher concentration of the ammonia strong solution always results in better cycle performance. Therefore, a saturation state is desired in the absorber. For saturated ammonia solution, when its temperature and pressure are fixed, its concentration is determined. No additional variable is added into the set of the free variables.

In the boiler, the leaving ammonia weak solution is bound to be saturated and the leaving ammonia vapor is in an equilibrium state with the weak solution. So when the 
pressure and temperature of the boiler, and the concentration of the feeding ammonia strong solution are fixed, the concentration of the weak solution and ammonia vapor along with their fractions are determined.

For heat source, there are three unknown variables: $m_{h s}, T_{h s}^{\text {in }}, T_{h s}^{\text {out }}$. However, due to the energy balance in the boiler and superheater, the energy transferred out of the heat source equals the energy transferred into the cycle. So only two variables are actually unknown. Any two of them can be chosen as free variables. We choose $T_{h s}^{i n}, T_{h s}^{\text {out }}$.

In summary, this ammonia-based combined power/cooling cycle has eight free

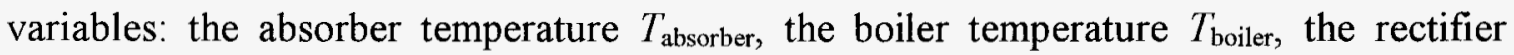

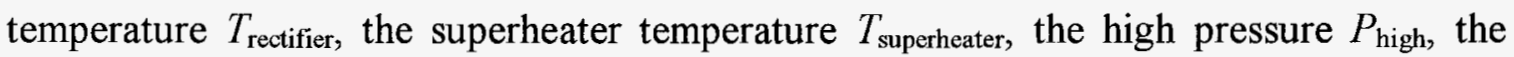
low pressure $P_{\text {low, }}$ the heat source entrance temperature $T_{h s}^{i n}$, and the heat source exit temperature $T_{h s}^{\text {out }}$. The cycle performance depends on the selection of the values for these eight free variables. Each combination of the eight values represents a distinctive operating condition of the cycle. Searching for optimum values for these variables is the task of this optimization work. Consequently, the objective function of the optimization of the cycle can be written as:

$$
\eta=f\left(T_{\text {absorber }}, T_{\text {boiler, }}, T_{\text {rectifier, }} T_{\text {superheater, }} P_{\text {high }}, P_{\text {low }}, T_{h s}^{\text {in }}, T_{h s}^{\text {out }}\right)
$$

As we have discussed above, second law thermal efficiency $\eta_{2}$ is the true reflection of the efficiency of resource utilization, it is chosen as our optimization objective.

After we have decided on the objective function, the next step is to find the constraints, which are required for the practicability of the cycle. These constraints define feasible region. By common sense, the temperature of the rectifier has to be lower than 
the temperatures of the boiler and superheater. Besides, the temperature of the boiler has to be greater than a minimum value, which is the bubble point of the feeding ammonia solution. Otherwise, no ammonia will be boiled off from the ammonia solution. Similarly, the temperature of the rectifier has to be greater than a minimum value so ammonia vapor entering the rectifier unit won't be totally condensed.

For the turbine, lowering its exhaust pressure will increase the thermal efficiency. However, the moisture content of the fluid leaving the turbine is increased at the same time. This increased moisture content decreases the efficiency of an actual turbine. In addition, the impingement of liquid droplet on the turbine blades leads to a serious erosion problem. In practice, it is desirable to keep the moisture content less than about 10 percent at the low pressure end of the turbine (Wark, 1988). Therefore, the quality of the ammonia vapor at the exit of the turbine has to be greater than $90 \%$.

In the cycle (shown in Figure 2.7), after being pumped to the high pressure, ammonia strong solution is separated into two streams (point 2). By common sense, their fractions are between 0 and 1 . Of course, when either of them is between 0 and 1 , the other must be. So only one constraint is added into the set of the constraints. The fraction of the solution at point 2 separated into the stream at point $2 ", f_{2}$, is randomly picked. In addition, the flow rate of the ammonia vapor leaving the boiler has to be less than that of feeding ammonia solution. Therefore, the fraction flowing through point $4, f_{4}$, which is defined by $m_{4} / m_{l}$, is between 0 and 1 .

Finally, the most common type of constraints in the thermodynamic cycle is the temperature difference limit on the heat exchangers. They are expressed mathematically with other constrains discussed as follows: 


$\begin{array}{ll}\text { - } & T_{\text {boiler }} \geq T_{\text {rectifier }} \\ \text { - } & T_{\text {superheater }} \geq T_{\text {rectifier }} \\ \text { - } & T_{\text {boiler }} \geq T_{\text {boilermin }} \\ \text { - } & T_{\text {rectifier }} \geq T_{\text {rectifiermin }} \\ \text { - } & x_{\text {turbine }} \geq 0.9 \\ \text { - } & 0 \leq f_{4} \leq 1 \\ \text { - } & T_{10}-T_{2}, \geq \Delta T_{\min } \\ \text { - } & T_{\mathrm{I}}-T_{\text {superheater }} \geq \Delta T_{\min } \\ \text { - } & T_{\mathrm{III}}-T_{3} \geq \Delta T_{\min } \\ \text { - } & T_{\mathrm{II}}-T_{\text {boiler }} \geq \Delta T_{\min } \\ & \Delta T_{\text {pin }} \geq \Delta T_{p i n}^{\min } \\ \text { - } & \end{array}$

Where

$x_{\text {turbine }}$ is the quality of the vapor at turbine exit;

$T_{\mathrm{x}}$ : temperature at point x (refer to Fig. 2.7);

$\Delta T_{\min }:$ minimum temperature difference required in the heat exchangers;

$\Delta T_{\text {pin }}:$ temperature difference at pinch point in the boiler;

$\Delta T_{p i n}^{\min }:$ minimum temperature difference required at pinch point.

The variables that appear in the constraints are free variables or the functions of free variables. Now, we have got an objective function and a complete set of constraints. The 
formulation of the optimization model for the ammonia-base combined power/cooling cycle is finished. Optimization work is ready to start.

\section{Optimization Program}

For optimization, we use the GRG2 program written by Dr. Lasdon. GRG2 is by far the most widely distributed nonlinear programming code (More, J. J., Wright, S. J., 1993). It uses an implementation of the generalized reduced gradient (GRG) algorithm. It has both Fortran and $\mathrm{C}$ version. $\mathrm{C}$ version is used in the current research. In the program, the user is required to provide a subroutine gcomp. The values of the objective function and constraints are calculated in this subroutine and provided to the main optimization program. The user also provides data specifying the upper and lower bounds on the free variables and initial values of the free variables. In the subroutine gcomp, the thermodynamic calculation of the ammonia-based combined power/cooling cycle is done to get the objective function, which is thermal efficiency, and constraints. Property programs are called to calculate the properties of ammonia/water mixture at various state points.

\section{Optimization Results}

In this section, optimization results are presented. Two typical heat source temperatures are studied. One is $440 \mathrm{~K}$, the other is $360 \mathrm{~K}$. The ambient temperatures for both cases are assumed as $290 \mathrm{~K}$. Their optimum working conditions are presented. 


\section{Resource Temperature of $440 K$}

This heat source temperature is within the range of geothermal source and a CPC solar collector. The cycle is optimized in terms of second law thermal efficiency. The optimum working conditions are presented in Table 4.1:

Table 4. 1 Optimum Working Conditions

\begin{tabular}{|c|c|c|c|c|c|c|}
\hline Point & $\mathrm{T}(\mathrm{K})$ & $\mathrm{P}(\mathrm{bar})$ & $\mathrm{h}(\mathrm{kJ} / \mathrm{kg})$ & $\mathrm{s}(\mathrm{kJ} / \mathrm{kg} . \mathrm{K})$ & $\mathrm{X}$ & $\begin{array}{c}\text { Flow Rate } \\
(\mathrm{kg} / \mathrm{s})\end{array}$ \\
\hline 1 & 295.0 & 8.6 & 66.0 & 0.3712 & 0.9500 & 1.0000 \\
\hline 2 & 296.1 & 52.0 & 72.9 & 0.3712 & 0.9500 & 1.0000 \\
\hline 3 & 313.4 & 52.0 & 156.2 & 0.6447 & 0.9500 & 1.0000 \\
\hline 4 & 374.3 & 52.0 & 1315.6 & 3.8000 & 0.9943 & 0.7720 \\
\hline 5 & 374.3 & 52.0 & 358.2 & 1.4403 & 0.7999 & 0.0000 \\
\hline 6 & 374.3 & 52.0 & 1315.6 & 3.8000 & 0.9943 & 0.7720 \\
\hline 7 & 435.0 & 52.0 & 1547.1 & 4.3606 & 0.9943 & 0.7720 \\
\hline 8 & 300.5 & 8.6 & 1270.9 & 4.3606 & 0.9943 & 0.7720 \\
\hline 9 & 300.5 & 8.6 & 1270.9 & 4.3606 & 0.9943 & 0.7720 \\
\hline 10 & 374.3 & 52.0 & 358.2 & 1.4403 & 0.7999 & 0.2280 \\
\hline 11 & 301.1 & 52.0 & -7.3 & 0.3568 & 0.7999 & 0.2280 \\
\hline 12 & 301.2 & 8.6 & -7.3 & 0.3775 & 0.7999 & 0.2280 \\
\hline
\end{tabular}

Boiler Heat Input:

Superheat Input:

Absorber Heat Rejection:

Turbine Work Output:

Vapor Quality at Turbine Exit:

Pump Work Input:

Total Heat Input:

Total Work Output:

First Law Efficiency:

Heat Source Flow Rate:

Heat Source Entrance Temperature:

Heat Source Exit Temperature:

Second law efficiency:
$941.1 \mathrm{~kJ} / \mathrm{s}$

$178.7 \mathrm{~kJ} / \mathrm{s}$

$913.5 \mathrm{~kJ} / \mathrm{s}$

$213.3 \mathrm{~kW}$

$97.55 \%$

$6.9 \mathrm{~kW}$

$1119.8 \mathrm{~kJ} / \mathrm{s}$

$206.37 \mathrm{~kW}$

$18.43 \%$

$2.783 \mathrm{~kg} / \mathrm{s}$

$440 \mathrm{~K}$

$345.4 \mathrm{~K}$

$59.63 \%$ 
It is noticed that the optimum concentration for ammonia strong solution is at 0.95. Though unnecessary, since the optimization program searches the entire concentration range to find the optimum concentration, another optimization is done at a fixed 0.9 concentration for ammonia strong solution to verify the previous optimization result. The result is given in Table 4.2 and shows that it has a lower second law efficiency.

Table 4.2 Optimum Working Conditions at $\mathrm{X}=0.9$

\begin{tabular}{|c|c|c|c|c|c|c|}
\hline Point & $\mathrm{T}(\mathrm{K})$ & $\mathrm{P}(\mathrm{bar})$ & $\mathrm{h}(\mathrm{kJ} / \mathrm{kg})$ & $\mathrm{s}(\mathrm{kJ} / \mathrm{kg} . \mathrm{K})$ & $\mathrm{X}$ & $\begin{array}{c}\text { Flow Rate } \\
(\mathrm{kg} / \mathrm{s})\end{array}$ \\
\hline 1 & 295.0 & 8.2 & 29.6 & 0.3438 & 0.9000 & 1.0000 \\
\hline 2 & 295.8 & 47.1 & 35.5 & 0.3438 & 0.9000 & 1.0000 \\
\hline 3 & 327.2 & 47.1 & 186.7 & 0.8293 & 0.9000 & 1.0000 \\
\hline 4 & 371.4 & 47.1 & 1329.4 & 3.8689 & 0.9939 & 0.5669 \\
\hline 5 & 371.4 & 47.1 & 326.4 & 1.3787 & 0.7771 & 0.0000 \\
\hline 6 & 371.4 & 47.1 & 1329.4 & 3.8689 & 0.9939 & 0.5669 \\
\hline 7 & 435.0 & 47.1 & 1559.5 & 4.4308 & 0.9939 & 0.5669 \\
\hline 8 & 302.3 & 8.2 & 1286.1 & 4.4308 & 0.9939 & 0.5669 \\
\hline 9 & 302.3 & 8.2 & 1286.1 & 4.4308 & 0.9939 & 0.5669 \\
\hline 10 & 371.4 & 47.1 & 326.4 & 1.3787 & 0.7771 & 0.4331 \\
\hline 11 & 300.8 & 47.1 & -22.6 & 0.3393 & 0.7771 & 0.4331 \\
\hline 12 & 301.0 & 8.2 & -22.6 & 0.3575 & 0.7771 & 0.4331 \\
\hline
\end{tabular}

Boiler Heat Input:

Superheat Input:

Absorber Heat Rejection:

Turbine Work Output:

Vapor Quality at Turbine Exit:

Pump Work Input:

Total Heat Input:

Total Work Output:

First Law Efficiency:

Heat Source Flow Rate:

Heat Source Entrance Temperature:

Heat Source Exit Temperature:

Second law efficiency:
$708.3 \mathrm{~kJ} / \mathrm{s}$

$130.4 \mathrm{~kJ} / \mathrm{s}$

$689.7 \mathrm{~kJ} / \mathrm{s}$

$155.0 \mathrm{~kW}$

$98.16 \%$

$5.9 \mathrm{~kW}$

$838.7 \mathrm{~kJ} / \mathrm{s}$

$149.07 \mathrm{~kW}$

$17.77 \%$

$2.072 \mathrm{~kg} / \mathrm{s}$

$440 \mathrm{~K}$

$344.8 \mathrm{~K}$

$57.86 \%$ 
At this heat source temperature, the optimum working conditions don't produce any cooling capacity. However, cooling capacity can be obtained at a non-optimum condition if necessary. Table 4.3 gives one of such conditions:

Table 4.3 A Working Condition with Cooling Capacity

\begin{tabular}{|c|c|c|c|c|c|c|}
\hline Point & $\mathrm{T}(\mathrm{K})$ & $\mathrm{P}(\mathrm{bar})$ & $\mathrm{h}(\mathrm{kJ} / \mathrm{kg})$ & $\mathrm{s}(\mathrm{kJ} / \mathrm{kg} . \mathrm{K})$ & $\mathrm{X}$ & $\begin{array}{c}\text { Flow Rate } \\
(\mathrm{kg} / \mathrm{s})\end{array}$ \\
\hline 1 & 295.0 & 4.6 & -125.9 & 0.1777 & 0.6131 & 1.0000 \\
\hline 2 & 295.2 & 31.8 & -122.4 & 0.1777 & 0.6131 & 1.0000 \\
\hline 3 & 374.8 & 31.8 & 334.2 & 1.5179 & 0.6131 & 1.0000 \\
\hline 4 & 403.0 & 31.8 & 1553.2 & 4.5993 & 0.9401 & 0.4078 \\
\hline 5 & 363.5 & 31.8 & 222.0 & 1.1859 & 0.6699 & 0.0648 \\
\hline 6 & 363.5 & 31.8 & 1377.5 & 4.1409 & 0.9911 & 0.3430 \\
\hline 7 & 366.4 & 31.8 & 1387.7 & 4.1684 & 0.9911 & 0.3430 \\
\hline 8 & 277.4 & 4.6 & 1139.2 & 4.1684 & 0.9911 & 0.3430 \\
\hline 9 & 285.0 & 4.6 & 1246.6 & 4.5514 & 0.9911 & 0.3430 \\
\hline 10 & 403.0 & 31.8 & 363.9 & 1.6145 & 0.4157 & 0.6570 \\
\hline 11 & 300.2 & 31.8 & -108.1 & 0.2668 & 0.4157 & 0.6570 \\
\hline 12 & 300.8 & 4.6 & -108.1 & 0.2774 & 0.4157 & 0.6570 \\
\hline
\end{tabular}

Boiler Heat Input:

Superheat Input:

Absorber Heat Rejection:

Turbine Work Output:

Vapor Quality at Turbine Exit:

Pump Work Input:

Cooling Capacity:

Total Heat Input:

Total Work Output:

First Law Efficiency:

Heat Source Flow Rate:

Heat Source Entrance Temperature:

Heat Source Exit Temperature:

Second law efficiency:
$523.9 \mathrm{~kJ} / \mathrm{s}$

$3.5 \mathrm{~kJ} / \mathrm{s}$

$482.5 \mathrm{~kJ} / \mathrm{s}$

$81.77 \mathrm{~kW}$

$90 \%$

$3.5 \mathrm{~kW}$

$36.8 \mathrm{~kW}$

$527.4 \mathrm{~kJ} / \mathrm{s}$

$81.77 \mathrm{~kW}$

$22.49 \%$

$2.211 \mathrm{~kg} / \mathrm{s}$

$440 \mathrm{~K}$

$384.4 \mathrm{~K}$

$43.13 \%$ 


\section{Resource Temperature of $360 \mathrm{~K}$}

This heat source temperature is within the range of flat-plate solar collectors and solar ponds. The cycle is optimized in terms of second law thermal efficiency. The optimum working condition for this heat source is listed in Table 4.4. At this heat source temperature, the optimum working condition does carry cooling capacity and the optimum concentration for ammonia strong solution is at a medium value, 0.67 .

Table 4.4 Optimum Working Condition

\begin{tabular}{|c|c|c|c|c|c|c|}
\hline Point & $\mathrm{T}(\mathrm{K})$ & $\mathrm{P}(\mathrm{bar})$ & $\mathrm{h}(\mathrm{kJ} / \mathrm{kg})$ & $\mathrm{s}(\mathrm{kJ} / \mathrm{kg} . \mathrm{K})$ & $\mathrm{X}$ & $\begin{array}{c}\text { Flow Rate } \\
(\mathrm{kg} / \mathrm{s})\end{array}$ \\
\hline 1 & 295.0 & 5.5 & -105.1 & 0.1989 & 0.6733 & 1.0000 \\
\hline 2 & 295.1 & 13.0 & -104.1 & 0.1989 & 0.6733 & 1.0000 \\
\hline 3 & 325.2 & 13.0 & 45.7 & 0.6805 & 0.6733 & 1.0000 \\
\hline 4 & 333.1 & 13.0 & 1371.9 & 4.4956 & 0.9935 & 0.2066 \\
\hline 5 & 311.1 & 13.0 & 102.9 & 0.5923 & 0.8949 & 0.0110 \\
\hline 6 & 311.1 & 13.0 & 1302.7 & 4.2793 & 0.9990 & 0.1956 \\
\hline 7 & 311.1 & 13.0 & 1302.7 & 4.2793 & 0.9990 & 0.1956 \\
\hline 8 & 280.6 & 5.5 & 1195.5 & 4.2793 & 0.9990 & 0.1956 \\
\hline 9 & 285.0 & 5.5 & 1278.3 & 4.5730 & 0.9990 & 0.1956 \\
\hline 10 & 333.1 & 13.0 & 45.2 & 0.7305 & 0.5942 & 0.8044 \\
\hline 11 & 300.1 & 13.0 & -106.8 & 0.2500 & 0.5942 & 0.8044 \\
\hline 12 & 300.2 & 5.5 & -106.8 & 0.2532 & 0.5942 & 0.8044 \\
\hline
\end{tabular}

Boiler Heat Input:

Superheat Input:

Absorber Heat Rejection:

Turbine Work Output:

Vapor Quality at Turbine Exit:

Pump Work Input:

Cooling Capacity:

Total Heat Input:

Total Work Output:

First Law Efficiency:
$272.9 \mathrm{~kJ} / \mathrm{s}$

$0 \mathrm{~kJ} / \mathrm{s}$

$269.1 \mathrm{~kJ} / \mathrm{s}$

$21.0 \mathrm{~kW}$

$93.93 \%$

$1.0 \mathrm{~kW}$

$16.2 \mathrm{~kW}$

$272.9 \mathrm{~kJ} / \mathrm{s}$

$19.99 \mathrm{~kW}$

$13.26 \%$ 
Heat Source Flow Rate:

$2.183 \mathrm{~kg} / \mathrm{s}$

Heat Source Entrance Temperature:

$360 \mathrm{~K}$

Heat Source Exit Temperature: $\quad 330.2 \mathrm{~K}$

Second law efficiency:

$54.22 \%$ 